\title{
ON THREE THIRD ORDER MOCK THETA FUNCTIONS AND HECKE-TYPE DOUBLE SUMS
}

\author{
ERIC MORTENSON
}

\begin{abstract}
We obtain four Hecke-type double sums for three of Ramanujan's third order mock theta functions. We discuss how these four are related to the new mock theta functions of Andrews' work on q-orthogonal polynomials and Bringmann, Hikami, and Lovejoy's work on unified Witten-Reshetikhin-Turaev invariants of certain Seifert manifolds. We then prove identities between these new mock theta functions by first expressing them in terms of the universal mock theta function.
\end{abstract}

\section{Notation}

Let $q$ be a nonzero complex number with $|q|<1$ and define $\mathbb{C}^{*}:=\mathbb{C}-\{0\}$. We recall some basics:

$$
\begin{gathered}
(x)_{n}=(x ; q)_{n}:=\prod_{i=0}^{n-1}\left(1-q^{i} x\right), \quad(x)_{\infty}=(x ; q)_{\infty}:=\prod_{i \geq 0}\left(1-q^{i} x\right), \\
j(x ; q):=(x)_{\infty}(q / x)_{\infty}(q)_{\infty}=\sum_{n}(-1)^{n} q^{\left(\begin{array}{c}
n \\
2
\end{array}\right)} x^{n}, \\
\text { and } j\left(x_{1}, x_{2}, \ldots, x_{n} ; q\right):=j\left(x_{1} ; q\right) j\left(x_{2} ; q\right) \cdots j\left(x_{n} ; q\right) .
\end{gathered}
$$

where in the last line the equivalence of product and sum follows from Jacobi's triple product identity. We also keep in mind the fact that $j\left(q^{n}, q\right)=0$ for $n \in \mathbb{Z}$. The following are special cases of the above definition. Here $a$ and $m$ are integers with $m$ positive. Define

$$
J_{a, m}:=j\left(q^{a} ; q^{m}\right), \quad J_{m}:=J_{m, 3 m}=\prod_{i \geq 1}\left(1-q^{m i}\right), \text { and } \bar{J}_{a, m}:=j\left(-q^{a} ; q^{m}\right) .
$$

\section{InTRODUCTION}

Historically, mock theta functions have many forms of representation: Eulerian forms, Hecke-type double sums, Appell-Lerch sums and Fourier coefficients of meromorphic Jacobi forms. Recently they have been cast as holomorphic parts of weak Maass forms. With the exception of a Hecke-type double sum for the third order mock theta function

Date: 18 January 2012.

2000 Mathematics Subject Classification. 11B65, 11F11, 11F27.

Key words and phrases. Hecke-type double sums, Appell-Lerch sums, mock theta functions, indefinite theta series. 
$\psi(q)$ found in Andrews' recent work on q-orthogonal polynomials [4], Hecke-type double sum representations for third order mock theta functions are unknown. Here we obtain Hecke-type sums for the third order functions $1+2 \psi(q), \nu(-q), \phi(q)$, and $\nu(q)$. Where these representations fit with respect to Zwegers' modularity theory [16] is also addressed. In the process, this leads us to two new mock theta functions found in Andrews' work [4] on $q$-orthogonal polynomials and to the two new mock theta functions found in Bringmann, Hikami, and Lovejoy's work [7] on unified Witten-Reshetikhin-Turaev invariants of certain Seifert manifolds. We obtain expressions for the new mock theta functions of [4, 7] in terms of the universal mock theta function

$$
g(x, q):=x^{-1}\left(-1+\sum_{n=0}^{\infty} \frac{q^{n^{2}}}{(x)_{n+1}(q / x)_{n}}\right)
$$

and use this information to prove identities between the new mock theta functions.

All of the results in this paper can be shown using Theorems 1.4 and 1.6 of [10], but for variety and brevity we will use other techniques on occasion.

We first recall some notation which will allow us to state Theorems 1.4 and 1.6 of [10]. We emphasize that it is Theorem 1.6 of [10] which guides us to representing the mock theta functions of [4, 7] in terms of $g(x, q)$. We will use the following definition of an Appell-Lerch sum:

Definition 1.1. Let $x, z \in \mathbb{C}^{*}$ with neither $z$ nor $x z$ an integral power of $q$. Then

$$
m(x, q, z):=\frac{1}{j(z ; q)} \sum_{r=-\infty}^{\infty} \frac{(-1)^{r} q^{\left(\begin{array}{l}
r \\
2
\end{array}\right)} z^{r}}{1-q^{r-1} x z} .
$$

In [10, Proposition 4.2], we showed that it is an easy consequence of [8, Theorem 2.2] that

$$
g(x, q)=-x^{-1} m\left(q^{2} x^{-3}, q^{3}, x^{2}\right)-x^{-2} m\left(q x^{-3}, q^{3}, x^{2}\right) .
$$

We recall the following notation for a special type of Hecke-type double sum:

Definition 1.2. Let $x, y \in \mathbb{C}^{*}$ and define $\operatorname{sg}(r):=1$ for $r \geq 0$ and $\operatorname{sg}(r):=-1$ for $r<0$. Then

$$
f_{a, b, c}(x, y, q):=\sum_{\operatorname{sg}(r)=\operatorname{sg}(s)} \operatorname{sg}(r)(-1)^{r+s} x^{r} y^{s} q^{a\left(\begin{array}{c}
r \\
2
\end{array}\right)+b r s+c\left(\begin{array}{c}
s \\
2
\end{array}\right)} .
$$

We also define the following expression involving Appell-Lerch sums:

$$
\begin{aligned}
g_{a, b, c}(x, & \left.y, q, z_{1}, z_{0}\right) \\
:= & \sum_{t=0}^{a-1}(-y)^{t} q^{c\left(\begin{array}{c}
t \\
2
\end{array}\right)} j\left(q^{b t} x ; q^{a}\right) m\left(-q^{a\left(\begin{array}{c}
b+1 \\
2
\end{array}\right)-c\left(\begin{array}{c}
a+1 \\
2
\end{array}\right)-t\left(b^{2}-a c\right)} \frac{(-y)^{a}}{(-x)^{b}}, q^{a\left(b^{2}-a c\right)}, z_{0}\right) \\
& +\sum_{t=0}^{c-1}(-x)^{t} q^{a\left(\begin{array}{c}
t \\
2
\end{array}\right)} j\left(q^{b t} y ; q^{c}\right) m\left(-q^{c\left(\begin{array}{c}
b+1 \\
2
\end{array}\right)-a\left(\begin{array}{c}
c+1 \\
2
\end{array}\right)-t\left(b^{2}-a c\right)} \frac{(-x)^{c}}{(-y)^{b}}, q^{c\left(b^{2}-a c\right)}, z_{1}\right) .
\end{aligned}
$$

We have 
ON THREE THIRD ORDER MOCK THETA FUNCTIONS AND HECKE-TYPE DOUBLE SUMS 3

Theorem 1.3 ([10], Theorem 1.6). Let $n$ and $p$ be positive integers with $(n, p)=1$. For generic $x, y \in \mathbb{C}^{*}$

$$
f_{n, n+p, n}(x, y, q)=g_{n, n+p, n}(x, y, q,-1,-1)+\theta_{n, p}(x, y, q)
$$

where

$$
\begin{aligned}
& \theta_{n, p}(x, y, q):=\sum_{r^{*}=0}^{p-1} \sum_{s^{*}=0}^{p-1} q^{n\left(\begin{array}{c}
r-(n-1) / 2 \\
2
\end{array}\right)+(n+p)(r-(n-1) / 2)(s+(n+1) / 2)+n\left(\begin{array}{c}
s+(n+1) / 2 \\
2
\end{array}\right)}(-x)^{r-(n-1) / 2} \\
& \cdot \frac{(-y)^{s+(n+1) / 2} J_{p^{2}(2 n+p)}^{3} j\left(-q^{n p(s-r)} x^{n} / y^{n} ; q^{n p^{2}}\right) j\left(q^{p(2 n+p)(r+s)+p(n+p)} x^{p} y^{p} ; q^{p^{2}(2 n+p)}\right)}{\bar{J}_{0, n p(2 n+p)} j\left(q^{p(2 n+p) r+p(n+p) / 2}(-y)^{n+p} /(-x)^{n}, q^{p(2 n+p) s+p(n+p) / 2}(-x)^{n+p} /(-y)^{n} ; q^{p^{2}(2 n+p)}\right)} .
\end{aligned}
$$

Here $r:=r^{*}+\{(n-1) / 2\}$ and $s:=s^{*}+\{(n-1) / 2\}$, with $0 \leq\{\alpha\}<1$ denoting the fractional part of $\alpha$.

We also have

Theorem 1.4 ([10], Theorem 1.4). Let $a, b$, and $c$ be positive integers with $a c<b^{2}$ and $b$ divisible by $a$ and $c$. Then

$$
f_{a, b, c}(x, y, q)=h_{a, b, c}(x, y, q,-1,-1)-\frac{1}{\bar{J}_{0, b^{2} / a-c} \bar{J}_{0, b^{2} / c-a}} \cdot \theta_{a, b, c}(x, y, q),
$$

where

$$
\begin{aligned}
h_{a, b, c}\left(x, y, q, z_{1}, z_{0}\right):=j( & \left.x ; q^{a}\right) m\left(-q^{a\left(\begin{array}{c}
b / a+1 \\
2
\end{array}\right)-c}(-y)(-x)^{-b / a}, q^{b^{2} / a-c}, z_{1}\right) \\
& +j\left(y ; q^{c}\right) m\left(-q^{c\left(\begin{array}{c}
b / c+1 \\
2
\end{array}\right)-a}(-x)(-y)^{-b / c}, q^{b^{2} / c-a}, z_{0}\right),
\end{aligned}
$$

and

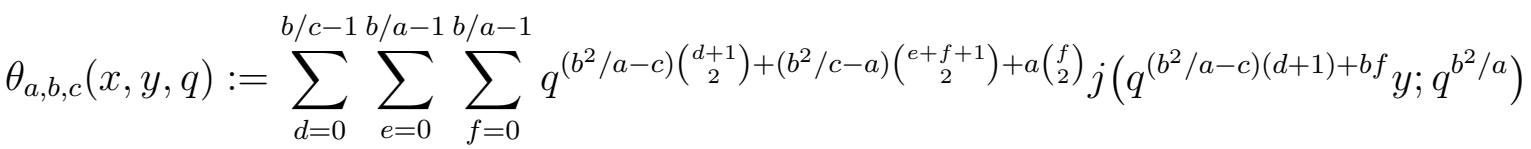

$$
\begin{aligned}
& \cdot(-x)^{f} j\left(q^{b\left(b^{2} /(a c)-1\right)(e+f+1)-\left(b^{2} / a-c\right)(d+1)+b^{3}(b-a) /\left(2 a^{2} c\right)}(-x)^{b / a} y^{-1} ; q^{\left(b^{2} / a\right)\left(b^{2} /(a c)-1\right)}\right) \\
& \cdot \frac{J_{b\left(b^{2} /(a c)-1\right)}^{3} j\left(q^{\left.\left(b^{2} / c-a\right)(e+1)+\left(b^{2} / a-c\right)(d+1)-c\left(\begin{array}{c}
b / c \\
2
\end{array}\right)-a\left(\begin{array}{c}
b / a \\
2
\end{array}\right)(-x)^{1-b / a}(-y)^{1-b / c} ; q^{b\left(b^{2} /(a c)-1\right)}\right)}\right.}{j\left(q^{\left(b^{2} / c-a\right)(e+1)-c\left(\begin{array}{c}
b / c \\
2
\end{array}\right)}(-x)(-y)^{-b / c}, q^{\left(b^{2} / a-c\right)(d+1)-a\left(\begin{array}{c}
b / a \\
2
\end{array}\right)}(-x)^{-b / a}(-y) ; q^{b\left(b^{2} /(a c)-1\right)}\right)} .
\end{aligned}
$$

Andrews [4, (1.10)] showed the following for Ramanujan's third order mock theta function $\psi(q)$ :

$$
1+\psi(q):=1+\sum_{n=1}^{\infty} \frac{q^{n^{2}}}{\left(q ; q^{2}\right)_{n}}=\frac{1}{(q)_{\infty}} \sum_{n=0}^{\infty}(-1)^{n} q^{2 n^{2}+n}\left(1-q^{6 n+6}\right) \sum_{j=0}^{n} q^{-\left(\begin{array}{c}
j+1 \\
2
\end{array}\right)} .
$$

This motivates 
Theorem 1.5. The third order mock theta functions $\psi(q)$ and $\nu(q)$ have the following Hecke-type double sum representations:

$$
\begin{aligned}
1+2 \psi(q) & =\frac{1}{(q)_{\infty}} \cdot \sum_{n=0}^{\infty}(-1)^{n} q^{2 n^{2}+n}\left(1+q^{2 n+1}\right) \sum_{j=-n}^{n} q^{-\left(\begin{array}{c}
j+1 \\
2
\end{array}\right)}, \\
\nu(-q) & =\frac{1}{(q)_{\infty}} \cdot \sum_{n=0}^{\infty}(-1)^{n} q^{2 n^{2}+2 n} \sum_{j=-n}^{n} q^{-\left(\begin{array}{c}
j+1 \\
2
\end{array}\right)} .
\end{aligned}
$$

In [4, (1.14), (1.15)], Andrews also showed the following for two new mock theta functions:

$$
\begin{gathered}
\bar{\psi}_{0}(q):=\sum_{n=0}^{\infty} \frac{q^{2 n^{2}}}{(-q ; q)_{2 n}}=\frac{1}{\left(q^{2} ; q^{2}\right)_{\infty}} \sum_{n=0}^{\infty} q^{4 n^{2}+n}\left(1-q^{6 n+3}\right) \sum_{j=-n}^{n}(-1)^{j} q^{-j^{2}} \\
\bar{\psi}_{1}(q):=\sum_{n=0}^{\infty} \frac{q^{2 n^{2}+2 n}}{(-q ; q)_{2 n+1}}=\frac{1}{\left(q^{2} ; q^{2}\right)_{\infty}} \sum_{n=0}^{\infty} q^{4 n^{2}+3 n}\left(1-q^{2 n+1}\right) \sum_{j=-n}^{n}(-1)^{j} q^{-j^{2}} .
\end{gathered}
$$

These four functions are related. Indeed, the four functions $1+2 \psi(q), \nu(-q), \bar{\psi}_{0}(q), \bar{\psi}_{1}(q)$ form a vector-valued mock theta function not unlike that for the fifth order mock theta functions $f_{0}(q), f_{1}(q), F_{0}(q), F_{1}(q)$ as found in [16]. It turns out that Andrews' two new mock theta functions can be written in terms of the third order mock theta function $\phi(q)$, where

$$
\phi(q):=\sum_{n=0}^{\infty} \frac{q^{n^{2}}}{\left(-q^{2} ; q^{2}\right)_{n}} .
$$

A straightforward exercise with Eulerian forms reveals that

$$
\begin{aligned}
2 \bar{\psi}_{0}\left(q^{2}\right) & =\phi(q)+\phi(-q), \\
2 q \bar{\psi}_{1}\left(q^{2}\right) & =\phi(q)-\phi(-q) .
\end{aligned}
$$

We note that the third order mock theta functions can all be written in terms of $g(x, q)$ [15]. In [8], Hickerson proved the mock theta conjectures. These are identities which express the fifth order mock theta functions in terms of the universal mock theta function $g(x, q)$ and theta functions. In [9], Hickerson found and then proved analogous identities for the seventh order functions. Here we prove similar identities for Andrews' two new mock theta functions of (1.7) and (1.8):

Theorem 1.6. The following identities are true:

$$
\begin{aligned}
& \bar{\psi}_{0}(q)=2-2 q g\left(-q, q^{8}\right)-\frac{J_{1,2} \bar{J}_{3,8}}{J_{2}}, \\
& \bar{\psi}_{1}(q)=2 q^{2} g\left(-q^{3}, q^{8}\right)+\frac{J_{1,2} \bar{J}_{1,8}}{J_{2}}
\end{aligned}
$$


We sketch how one is led to such identities. Once one has the Hecke form of the mock theta function, one uses Theorem 1.3 and basic Appell-Lerch sum properties as a guide to produce an expression like identity (1.3). In the process, it is best to ignore the theta functions as well as the $z$ part of the $m(x, q, z)$ terms. What is left is a theta function, so one uses a software package such as Maple or Mathematica to determine if the theta function has a nice form. For both $\bar{\psi}_{0}(q)$ and $\bar{\psi}_{1}(q)$, that is the case.

Vector-valued mock theta functions tend to come in pairs. The above four functions can all be written in terms of $f_{3,5,3}(x, y, q)$ 's, so [16] suggests that the paired vector might consist of functions which can be written in terms of $f_{1,7,1}(x, y, q)$ 's. How one goes about finding such a pair is not obvious. Sometimes, mock theta functions are sign flips away from a theta function. So with this in mind, we recall the following identity which is found in Andrews [2, (1.2)] as well as Kac and Peterson [11, (5.19)]:

$$
\begin{aligned}
J_{1}^{2} & =\sum_{n=0}^{\infty} q^{2 n^{2}+n}\left(1-q^{2 n+1}\right) \sum_{j=-n}^{n}(-1)^{j} q^{-3 j^{2} / 2+j / 2} \\
& =\left(\sum_{\substack{n+j \geq 0 \\
n-j \geq 0}}-\sum_{\substack{n+j<0 \\
n-j<0}}\right)(-1)^{j} q^{2 n^{2}+n-3 j^{2} / 2+j / 2}=f_{1,7,1}\left(q, q^{2}, q\right)-q f_{1,7,1}\left(q^{3}, q^{4}, q\right) .
\end{aligned}
$$

Making some judicious sign flips, we find that

$$
\begin{aligned}
\bar{J}_{1,4} \cdot \phi(q) & =\sum_{n=0}^{\infty}(-1)^{n} q^{2 n^{2}+n}\left(1+q^{2 n+1}\right) \sum_{j=-n}^{n}(-1)^{j} q^{-3 j^{2} / 2+j / 2} \\
& =\left(\sum_{\substack{n+j \geq 0 \\
n-j \geq 0}}-\sum_{\substack{n+j<0 \\
n-j<0}}\right)(-1)^{n+j} q^{2 n^{2}+n-3 j^{2} / 2+j / 2} \\
& =f_{1,7,1}\left(-q,-q^{2}, q\right)+q f_{1,7,1}\left(-q^{3},-q^{4}, q\right) .
\end{aligned}
$$

To find the other components of the vector-valued mock theta function, Zwegers' thesis [16] leads us to

$$
\begin{aligned}
\bar{J}_{1,4} \cdot \nu(q) & =\sum_{n=0}^{\infty}(-1)^{n} q^{2 n^{2}+2 n} \sum_{j=-n}^{n}(-1)^{j} q^{-3 j^{2} / 2+j / 2} \\
& =\frac{1}{2}\left(\sum_{\substack{n+j \geq 0 \\
n-j \geq 0}}-\sum_{\substack{n+j<0 \\
n-j<0}}\right)(-1)^{n+j} q^{2 n^{2}+2 n-3 j^{2} / 2+j / 2}, \\
J_{1,2} \cdot\left(q \bar{\phi}_{0}(q)+1\right) & =\sum_{n=0}^{\infty} q^{4 n^{2}+n}\left(1-q^{6 n+3}\right) \sum_{j=-n}^{n}(-1)^{j} q^{-3 j^{2}-j} \\
& =\left(\sum_{\substack{n+j \geq 0 \\
n-j \geq 0}}-\sum_{\substack{n+j<0 \\
n-j<0}}\right)(-1)^{j} q^{4 n^{2}+n-3 j^{2}-j},
\end{aligned}
$$




$$
\begin{aligned}
J_{1,2} \cdot \bar{\phi}_{1}(q) & =\sum_{n=0}^{\infty} q^{4 n^{2}+3 n}\left(1-q^{2 n+1}\right) \sum_{j=-n}^{n}(-1)^{j} q^{-3 j^{2}-j} \\
& =\left(\sum_{\substack{n+j \geq 0 \\
n-j \geq 0}}-\sum_{\substack{n+j<0 \\
n-j<0}}\right)(-1)^{j} q^{4 n^{2}+3 n-3 j^{2}-j}
\end{aligned}
$$

The last two are two new mock theta functions of Bringmann, Hikami, and Lovejoy [7], where

$$
\bar{\phi}_{0}(q):=\sum_{n=0}^{\infty} q^{n}(-q)_{2 n+1} \text { and } \bar{\phi}_{1}(q):=\sum_{n=0}^{\infty} q^{n}(-q)_{2 n} .
$$

We also note that (1.16) is a slightly rewritten [7, (2.7)]. We will prove

Theorem 1.7. Identities 1.14) - 1.17) are true.

We will also prove identities between Andrews' two new mock theta functions and Bringmann, Hikami, and Lovejoy's new mock theta functions. We recall that Bringmann, Hikami, and Lovejoy also proved

$$
\begin{aligned}
2 q^{2} \bar{\phi}_{0}\left(q^{2}\right) & =\psi(q)+\psi(-q), \\
2 q \bar{\phi}_{1}\left(q^{2}\right) & =\psi(q)-\psi(-q) .
\end{aligned}
$$

We express $\bar{\phi}_{0}(q)$ and $\bar{\phi}_{1}(q)$ in terms of $g(x, q)$.

Theorem 1.8. The following identities are true:

$$
\begin{gathered}
q \bar{\phi}_{0}(q)=-1+q g\left(-q, q^{8}\right)+\frac{\bar{J}_{2,4} \bar{J}_{3,8}}{J_{2}} \\
\bar{\phi}_{1}(q)=-q^{2} g\left(-q^{3}, q^{8}\right)+\frac{\bar{J}_{2,4} \bar{J}_{1,8}}{J_{2}}
\end{gathered}
$$

The above two identities were found using Theorem 1.3 as a guide. Here the respective Hecke-type forms are in terms of $f_{1,7,1}(x, y, q)$ 's. We could use Theorem 1.3 to prove these two identities, but for brevity, we will use new results which follow from Appell-Lerch sum properties of [10].

Using Theorems [1.6 and 1.8, we then have the following immediate corollary which relates the mock theta functions of Andrews [4] to those of Bringmann, Hikami, and Lovejoy [7].

Corollary 1.9. The following identities are true:

$$
\begin{gathered}
\bar{\psi}_{0}(q)+2 q \bar{\phi}_{0}(q)=-\frac{\bar{J}_{3,8}}{J_{2}} \cdot\left(J_{1,2}-2 \bar{J}_{2,4}\right), \\
\bar{\psi}_{1}(q)+2 \bar{\phi}_{1}(q)=\frac{\bar{J}_{1,8}}{J_{2}} \cdot\left(J_{1,2}+2 \bar{J}_{2,4}\right) .
\end{gathered}
$$


In Section 2, we recall useful facts covering theta function identities, Appell-Lerch sum properties, Hecke-type double sums, and third order mock theta functions. We also recall and give new proofs of properties found in 14 for the universal mock theta function $g(x, q)$. In Section 3, we prove Theorem 1.5. Here we prove identity (1.5) with Theorem 1.3. We could use Theorem 1.3 to prove identity (1.6), but for variety, we use a different technique. One could also use Corollary 6 of [4]. Theorem 1.6 is shown in Section 4. Here we use Theorem [1.3 for both identities. In Section [5 we prove Theorem 1.7. For identities (1.14) and (1.15) we rewrite the Hecke-type doube sums and use Theorem 1.4. For identities (1.16) and (1.17) we use the Bailey pair techniques of [3]. In Section 6, we prove Theorem 1.8 .

\section{Preliminaries}

2.1. Properties of theta functions. For later use, we state the following easily shown identities:

$$
\begin{gathered}
\bar{J}_{0,1}=2 \bar{J}_{1,4}=\frac{2 J_{2}^{2}}{J_{1}}, \bar{J}_{1,2}=\frac{J_{2}^{5}}{J_{1}^{2} J_{4}^{2}}, J_{1,2}=\frac{J_{1}^{2}}{J_{2}}, \bar{J}_{1,3}=\frac{J_{2} J_{3}^{2}}{J_{1} J_{6}}, \\
J_{1,4}=\frac{J_{1} J_{4}}{J_{2}}, J_{1,6}=\frac{J_{1} J_{6}^{2}}{J_{2} J_{3}}, \bar{J}_{1,6}=\frac{J_{2}^{2} J_{3} J_{12}}{J_{1} J_{4} J_{6}} .
\end{gathered}
$$

We state additional identities:

$$
\begin{gathered}
j\left(q^{n} x ; q\right)=(-1)^{n} q^{-\left(\begin{array}{c}
n \\
2
\end{array}\right)} x^{-n} j(x ; q), \quad n \in \mathbb{Z}, \\
j(x ; q)=j(q / x ; q)=-x j\left(x^{-1} ; q\right), \\
j(-x ; q)=J_{1,2} j\left(x^{2} ; q^{2}\right) / j(x ; q) \text { if } x \text { is not an integral power of } q, \\
j(x ; q)=J_{1} j\left(x, q x, \ldots, q^{n-1} x ; q^{n}\right) / J_{n}^{n} \text { if } n \geq 1, \\
j(x ;-q)=j\left(x ; q^{2}\right) j\left(-q x ; q^{2}\right) / J_{1,4}, \\
j(z ; q)=\sum_{k=0}^{m-1}(-1)^{k} q^{\left(\begin{array}{c}
k \\
2
\end{array}\right) z^{k} j\left((-1)^{m+1} q^{\left(\begin{array}{c}
m \\
2
\end{array}\right)+m k} z^{m} ; q^{m^{2}}\right),} \\
j\left(x^{n} ; q^{n}\right)=J_{n} j\left(x, \zeta_{n} x, \cdots, \zeta_{n}^{n-1} x ; q^{n}\right) / J_{1}^{n} \text { if } n \geq 1
\end{gathered}
$$

where and $\zeta_{n}$ an $n$-th primitive root of unity. We recall the classical partial fraction expansion for the reciprocal of Jacobi's theta product

$$
\sum_{n} \frac{(-1)^{n} q^{\left(\begin{array}{c}
n+1 \\
2
\end{array}\right)}}{1-q^{n} z}=\frac{J_{1}^{3}}{j(z ; q)},
$$

where $z$ is not an integral power of $q$. A convenient form of the Riemann relation for theta functions is

Proposition 2.1. For generic $a, b, c, d \in \mathbb{C}^{*}$

$$
j(a c, a / c, b d, b / d ; q)=j(a d, a / d, b c, b / c ; q)+b / c \cdot j(a b, a / b, c d, c / d ; q) .
$$

We collect several useful results about theta functions in terms of a proposition: 
Proposition 2.2. For generic $x, y, z \in \mathbb{C}^{*}$

$$
\begin{gathered}
j\left(q x^{3} ; q^{3}\right)+x j\left(q^{2} x^{3} ; q^{3}\right)=j(-x ; q) j\left(q x^{2} ; q^{2}\right) / J_{2}=J_{1} j\left(x^{2} ; q\right) / j(x ; q), \\
j(x ; q) j(y ; q)=j\left(-x y ; q^{2}\right) j\left(-q x^{-1} y ; q^{2}\right)-x j\left(-q x y ; q^{2}\right) j\left(-x^{-1} y ; q^{2}\right), \\
j(-x ; q) j(y ; q)-j(x ; q) j(-y ; q)=2 x j\left(x^{-1} y ; q^{2}\right) j\left(q x y ; q^{2}\right), \\
j(-x ; q) j(y ; q)+j(x ; q) j(-y ; q)=2 j\left(x y ; q^{2}\right) j\left(q x^{-1} y ; q^{2}\right),
\end{gathered}
$$

Identity (2.3a) is the quintuple product identity.

Finally, we recall a fact which follows immediately from [6, Lemma 2] and is also [8, Theorem 1.7].

Proposition 2.3. Let $C$ be a nonzero complex number, and let $n$ be a nonnegative integer. Suppose that $F(z)$ is analytic for $z \neq 0$ and satisfies $F(q z)=C z^{-n} F(z)$. Then either $F(z)$ has exactly $n$ zeros in the annulus $|q|<|z| \leq 1$ or $F(z)=0$ for all $z$.

We will need the following four identities, which appear to be new.

Proposition 2.4. Let $x \neq 0$. Then

$$
j\left(q^{2} x ; q^{4}\right) j\left(q^{5} x ; q^{8}\right)+\frac{q}{x} \cdot j\left(x ; q^{4}\right) j\left(q x ; q^{8}\right)-\frac{J_{1}}{J_{4}} \cdot j\left(-q^{3} x ; q^{4}\right) j\left(q^{3} x ; q^{8}\right)=0
$$

Proof of Proposition 2.4. Let $f(x)$ be the left hand side of (2.4). It satisfies $f\left(q^{8} x\right)=$ $-q^{-13} x^{-3} f(x)$. By Proposition 2.3, if $f$ has more than 3 zeros in $\left|q^{8}\right|<|x| \leq 1$, then $f(x)=0$ for all nonzero $x$. But it is easy to check that $f(x)=0$ for $x=1, q^{2}, q^{3}, q^{7}$.

Proposition 2.5. Let $x \neq 0$. Then

$$
J_{12}^{3} j\left(q^{2} x ; q^{3}\right) j\left(-q x^{2} ; q^{6}\right)+x J_{12}^{3} j\left(q x ; q^{3}\right) j\left(-q^{5} x^{2} ; q^{6}\right)-J_{2,12}^{2} J_{4} j\left(-x ; q^{3}\right) j\left(q^{3} x^{2} ; q^{6}\right)=0
$$

Proof of Proposition 2.5. Let $f(x)$ be the left hand side of (2.5). It satisfies $f\left(q^{3} x\right)=$ $-q^{-3} x^{-3} f(x)$. By Proposition [2.3, if $f$ has more than 3 zeros in $\left|q^{3}\right|<|x| \leq 1$, then $f(x)=0$ for all nonzero $x$. But it is easy to check that $f(x)=0$ for $x=-1, \pm i q^{1 / 2}, q$, $\pm q^{3 / 2}, q^{2}, \pm q^{5 / 2}$.

Proposition 2.6. We have

$$
\begin{aligned}
J_{3,6} J_{2,16}^{2}-J_{4,8} J_{3,24} J_{1,8} & =q J_{1,2} J_{2,8} \bar{J}_{24,96}, \\
-J_{3,6} J_{6,16}^{2}+J_{4,8} J_{9,24} J_{3,8} & =q^{3} J_{1,2} J_{2,8} \bar{J}_{24,96 .} .
\end{aligned}
$$

To prove these identities, we first give a lemma.

Lemma 2.7. Let $z \neq 0$. Then

$$
\begin{gathered}
J_{12,24} j\left(q^{4} z^{4} ; q^{8}\right)-j\left(q^{3} z^{3} ; q^{6}\right) j\left(-q^{4} z^{2} ; q^{8}\right)-q^{3} z^{-3} j\left(q z ; q^{2}\right) j\left(-z^{6} ; q^{24}\right)=0, \quad \\
J_{2,8} j\left(q^{12} z^{2} ; q^{24}\right)-\bar{J}_{2,8} j\left(q^{3} z ; q^{6}\right)+q^{2} j\left(q z ; q^{8}\right) j\left(q^{3} z^{-1} ; q^{24}\right)+q^{2} j\left(q z^{-1} ; q^{8}\right) j\left(q^{3} z ; q^{24}\right)=0 .
\end{gathered}
$$


Proof of Lemma 2.7. We prove (2.8). Let $f(z)$ be the left hand side of (2.8). It satisfies $f\left(q^{4} z\right)=q^{-16} z^{-8} f(z)$. By Proposition [2.3, if $f$ has more than 8 zeros in $\left|q^{4}\right|<|z| \leq 1$, then $f(z)=0$ for all nonzero $z$. The first term in (2.8) is zero for $z= \pm q, \pm i q, \pm q^{3}$, $\pm i q^{3}$, and the second term is zero for $z=i q^{2}$ (among others) so checking that $f(z)=0$ for these 9 values just involves product rearrangements of the other two terms. Here we use facts such as $j(i, q)=(1-i) J_{1,4}$ and $j\left(i q, q^{2}\right)=J_{4,8}$.

We prove (2.9). Let $f(z)$ be the left side of (2.9); it satisfies $f\left(q^{24}\right) z=q^{-48} z^{-4} f(z)$. So if it has more than 4 zeros in $\left|q^{24}\right|<|z| \leq 1$, then $f(z)=0$ for all $z$. It is easy to see that $f(z)=0$ for $z=q^{3}, q^{9}, q^{15}$, and $q^{21}$; in each case the second term and one of the last two terms in $f(z)$ is zero. Also,

$$
\begin{aligned}
f(q) & =J_{2,8} J_{10,24}-\bar{J}_{2,8} J_{2}+q^{2} J_{2,8} J_{2,24}+0=J_{2,8}\left(J_{10,24}+q^{2} J_{2,24}\right)-\bar{J}_{2,8} J_{2} \\
& =J_{2,8} j\left(-q^{2} ;-q^{6}\right)-\bar{J}_{2,8} J_{2}=0 .
\end{aligned}
$$

So $f(z)=0$ for all $z$.

Proof of Proposition 2.6. We will use the following two identities

$$
\begin{aligned}
2 q^{2} J_{2,16}^{2} & =\bar{J}_{2,8} J_{4,8}-J_{2,8} \bar{J}_{4,8}, \\
2 J_{6,16}^{2} & =\bar{J}_{2,8} J_{4,8}+J_{2,8} \bar{J}_{4,8},
\end{aligned}
$$

which follow respectively from (2.3c) and (2.3d) with $q \rightarrow q^{8}, x=q^{2}, y=q^{4}$.

We prove (2.6). Letting $z=1$ in (2.8) gives

$$
0=J_{12,24} J_{4,8}-J_{3,6} \bar{J}_{4,8}-q^{3} J_{1,2} \bar{J}_{0,24}=J_{12,24} J_{4,8}-J_{3,6} \bar{J}_{4,8}-2 q^{3} J_{1,2} \bar{J}_{24,96} .
$$

Multiply by $J_{2,8}$ and rearrange to obtain

$$
2 q^{3} J_{2,8} J_{1,2} \bar{J}_{24,96}=J_{2,8} J_{12,24} J_{4,8}-J_{3,6} J_{2,8} \bar{J}_{4,8} \text {. }
$$

Letting $z=1$ in (2.9) gives

$$
J_{2,8} J_{12,24}-\bar{J}_{2,8} J_{3,6}+2 q^{2} J_{1,8} J_{3,24}=0 .
$$

Multiply by $J_{4,8}$ and rearrange to obtain

$$
J_{2,8} J_{12,24} J_{4,8}=J_{3,6} \bar{J}_{2,8} J_{4,8}-2 q^{2} J_{1,8} J_{3,24} J_{4,8} .
$$

Substitute (2.15) into (2.13) and use (2.10):

$$
\begin{aligned}
2 q^{3} J_{2,8} J_{1,2} \bar{J}_{24,96} & =J_{3,6} \bar{J}_{2,8} J_{4,8}-2 q^{2} J_{1,8} J_{3,24} J_{4,8}-J_{3,6} J_{2,8} \bar{J}_{4,8} \\
& =J_{3,6} 2 q^{2} J_{2,16}^{2}-2 q^{2} J_{1,8} J_{3,24} J_{4,8} .
\end{aligned}
$$

Dividing by $2 q^{2}$ yields (2.6).

We prove (2.7). Setting $z=q^{12}$ in (2.9), we have

$$
J_{2,8} J_{36,24}-\bar{J}_{2,8} J_{15,6}+q^{2} J_{13,8} J_{-9,24}+q^{2} J_{-11,8} J_{15,25}=0,
$$

which can be rewritten

$$
2 J_{3,8} J_{9,24}=J_{2,8} J_{12,24}+\bar{J}_{2,8} J_{3,6} .
$$


Multiply by $J_{4,8}$ and rearrange to obtain

$$
J_{2,8} J_{12,24} J_{4,8}=-J_{3,6} \bar{J}_{2,8} J_{4,8}+2 J_{3,8} J_{9,24} J_{4,8} .
$$

Substitute this into (2.13) and use (2.11):

$$
\begin{aligned}
2 q^{3} J_{2,8} J_{1,2} \bar{J}_{24,96} & =-J_{3,6} \bar{J}_{2,8} J_{4,8}+2 J_{3,8} J_{9,24} J_{4,8}-J_{3,6} J_{2,8} \bar{J}_{4,8} \\
& =-J_{3,6} 2 J_{6,16}^{2}+2 J_{3,8} J_{9,24} J_{4,8} .
\end{aligned}
$$

Dividing by 2 gives (2.7).

2.2. Properties of the Appell-Lerch sums. The Appell-Lerch sum $m(x, q, z)$ satisfies several functional equations and identities, which we collect in the form of a proposition.

Proposition 2.8. For generic $x, z, z_{0}, z_{1} \in \mathbb{C}^{*}$

$$
\begin{gathered}
m(x, q, z)=m(x, q, q z), \\
m(x, q, z)=x^{-1} m\left(x^{-1}, q, z^{-1}\right), \\
m(q x, q, z)=1-x m(x, q, z), \\
m(x, q, z)=1-q^{-1} x m\left(q^{-1} x, q, z\right), \\
m(x, q, z)=x^{-1}-x^{-1} m(q x, q, z), \\
m\left(x, q, z_{1}\right)-m\left(x, q, z_{0}\right)=\frac{z_{0} J_{1}^{3} j\left(z_{1} / z_{0} ; q\right) j\left(x z_{0} z_{1} ; q\right)}{j\left(z_{0} ; q\right) j\left(z_{1} ; q\right) j\left(x z_{0} ; q\right) j\left(x z_{1} ; q\right)},
\end{gathered}
$$

The proofs are straightforward and will be omitted. Although one can find most of these in [12, 13], these papers are hard to obtain. In addition, the German summary [13] has a few typos. The equivalent of (2.19f), for example, reads

$$
m\left(x, q, z_{1}\right)=m\left(x, q, z_{0}\right)=\frac{z_{0} J_{1}^{3} j\left(z_{1} / z_{0} ; q\right) j\left(x z_{0} z_{1} ; q\right)}{j\left(z_{0} ; q\right) j\left(z_{1} ; q\right) j\left(x z_{0} ; q\right) j\left(x z_{1} ; q\right)} .
$$

A modern list of Appell-Lerch sum properties with proofs can be found in [16].

We recall a useful result:

Theorem 2.9 ([10], Theorem 3.6). For generic $x, z, z^{\prime} \in \mathbb{C}^{*}$

$$
\begin{aligned}
& m(x, q, z)=\sum_{r=0}^{n-1} q^{-\left(\begin{array}{c}
r+1 \\
2
\end{array}\right)}(-x)^{r} m\left(-q^{\left(\begin{array}{c}
n \\
2
\end{array}\right)-n r}(-x)^{n}, q^{n^{2}}, z^{\prime}\right) \\
& \quad+\frac{z^{\prime} J_{n}^{3}}{j(x z ; q) j\left(z^{\prime} ; q^{n^{2}}\right)} \sum_{r=0}^{n-1} \frac{q^{\left(\begin{array}{c}
r \\
2
\end{array}\right)}(-x z)^{r} j\left(-q^{\left(\begin{array}{c}
n \\
2
\end{array}\right)+r}(-x)^{n} z z^{\prime} ; q^{n}\right) j\left(q^{n r} z^{n} / z^{\prime} ; q^{n^{2}}\right)}{j\left(-q^{\left(\begin{array}{c}
n \\
2
\end{array}\right)}(-x)^{n} z^{\prime}, q^{r} z ; q^{n}\right)} .
\end{aligned}
$$

Identity (2.1a) easily yields two $n=2$ specializations: 
Corollary 2.10. For generic $x, z, z^{\prime} \in \mathbb{C}^{*}$

$$
\begin{aligned}
& m(x, q, z)=m\left(-q x^{2}, q^{4}, z^{\prime}\right)-q^{-1} x m\left(-q^{-1} x^{2}, q^{4}, z^{\prime}\right) \\
& \quad+\frac{z^{\prime} J_{2}^{3}}{j(x z ; q) j\left(z^{\prime} ; q^{4}\right)}\left[\frac{j\left(-q x^{2} z z^{\prime} ; q^{2}\right) j\left(z^{2} / z^{\prime} ; q^{4}\right)}{j\left(-q x^{2} z^{\prime}, z ; q^{2}\right)}-x z \frac{j\left(-q^{2} x^{2} z z^{\prime} ; q^{2}\right) j\left(q^{2} z^{2} / z^{\prime} ; q^{4}\right)}{j\left(-q x^{2} z^{\prime}, q z ; q^{2}\right)}\right] .
\end{aligned}
$$

Corollary 2.11. For generic $x, z \in \mathbb{C}^{*}$

$$
\begin{aligned}
m(x, q, z)= & m\left(-q x^{2}, q^{4},-1\right)-q^{-1} x m\left(-q^{-1} x^{2}, q^{4},-1\right) \\
& -\frac{J_{2}^{3}}{j(x z ; q) j\left(q x^{2} ; q^{2}\right) \bar{J}_{0,4}}\left[\frac{j\left(q x^{2} z ; q^{2}\right) j\left(-z^{2} ; q^{4}\right)}{j\left(z ; q^{2}\right)}-x z \frac{j\left(q^{2} x^{2} z ; q^{2}\right) j\left(-q^{2} z^{2} ; q^{4}\right)}{j\left(q z ; q^{2}\right)}\right] .
\end{aligned}
$$

We recall an identity [10, Proposition 4.2], [8, Theorem 2.2], which expresses the universal mock theta function in terms of Appell-Lerch sums:

Proposition 2.12. For generic $x, z \in \mathbb{C}^{*}$

$$
g(x, q)=-x^{-2} m\left(q x^{-3}, q^{3}, x^{3} z\right)-x^{-1} m\left(q^{2} x^{-3}, q^{3}, x^{3} z\right)+\frac{J_{1}^{2} j(x z ; q) j\left(z ; q^{3}\right)}{j(x ; q) j(z ; q) j\left(x^{3} z ; q^{3}\right)} .
$$

Taking the limit $z \rightarrow 1$ yields the following corollary:

Corollary 2.13. For generic $x \in \mathbb{C}^{*}$

$$
g(x, q)=-x^{-1} m\left(q^{2} x^{-3}, q^{3}, x^{3}\right)-x^{-2} m\left(q x^{-3}, q^{3}, x^{3}\right)+\frac{J_{3}^{3}}{J_{1} j\left(x^{3} ; q^{3}\right)} .
$$

The following identity for $g(x, q)$ can be found in the lost notebook.

Proposition 2.14. [14, p. 32], [5, (12.5.3)] For generic $x$

$$
g(x, q)=-x^{-1}+q x^{-3} g\left(-q x^{-2}, q^{4}\right)-q g\left(-q x^{2}, q^{4}\right)+\frac{J_{2} J_{2,4}^{2}}{x j(x ; q) j\left(-q x^{2} ; q^{2}\right)} .
$$

Proposition 2.14 has a useful and easily shown corollary, the first half of which is also in the lost notebook [14, p. 39], [5, (12.4.4)].

Corollary 2.15. For generic $x \in \mathbb{C}$

$$
\begin{aligned}
& g(x, q)+g(-x, q)=-2 q g\left(-q x^{2}, q^{4}\right)+\frac{2 J_{2} \bar{J}_{1,4}^{2}}{j\left(-q x^{2} ; q^{4}\right) j\left(x^{2} ; q^{2}\right)}, \\
& g(x, q)-g(-x, q)=-2 x^{-1}+2 q x^{-3} g\left(-q x^{-2}, q^{4}\right)+\frac{2 J_{2} \bar{J}_{1,4}^{2}}{x j\left(-q^{3} x^{2} ; q^{4}\right) j\left(x^{2} ; q^{2}\right)} .
\end{aligned}
$$

We give a new proof of Proposition 2.14.

Proof of Proposition 2.14. We note the easily shown identity

$$
g(x, q)=g(q / x, q)
$$


Applying Corollary (2.10) with $z^{\prime}=q^{6} x^{4}$ to each Appell-Lerch sum of (1.3), we have

$$
\begin{aligned}
g(x, q)= & -x^{-1} m\left(q^{2} x^{-3}, q^{3}, x^{2}\right)-x^{-2} m\left(q x^{-3}, q^{3}, x^{2}\right) \\
= & -x^{-1} m\left(-q^{7} x^{-6}, q^{12}, q^{6} x^{4}\right)+q^{-1} x^{-4} m\left(-q x^{-6}, q^{12}, q^{6} x^{4}\right) \\
& \quad-\frac{q^{6} x^{3} J_{6}^{3}}{j\left(q^{2} x^{-1} ; q^{3}\right) j\left(-q^{13} x^{-2} ; q^{6}\right) j\left(q^{6} x^{4} ; q^{12}\right)} \cdot \frac{\bar{J}_{13,6} j\left(q^{-6} ; q^{12}\right)}{j\left(x^{2} ; q^{6}\right)} \\
& -x^{-2} m\left(-q^{5} x^{-6}, q^{12}, q^{6} x^{4}\right)+q^{-2} x^{-5} m\left(-q^{-1} x^{-6}, q^{12}, q^{6} x^{4}\right) \\
& -\frac{q^{6} x^{2} J_{6}^{3}}{j\left(q x^{-1} ; q^{3}\right) j\left(-q^{11} x^{-2} ; q^{6}\right) j\left(q^{6} x^{4} ; q^{12}\right)} \cdot \frac{\bar{J}_{11,6} j\left(q^{-6} ; q^{12}\right)}{j\left(x^{2} ; q^{6}\right)} .
\end{aligned}
$$

Using (1.3), we also have

$$
\begin{aligned}
-q g\left(-q x^{2}, q^{4}\right) & =-q g\left(-q^{3} x^{-2}, q^{4}\right) \\
& =-q^{-2} x^{2} m\left(-q^{-1} x^{6}, q^{12}, q^{6} x^{-4}\right)+q^{-5} x^{4} m\left(-q^{-5} x^{6}, q^{12}, q^{6} x^{-4}\right) \\
& =q^{-1} x^{-4} m\left(-q x^{-6}, q^{12}, q^{6} x^{4}\right)-x^{-2} m\left(-q^{5} x^{-6}, q^{12}, q^{6} x^{4}\right),
\end{aligned}
$$

where the last line follows from (2.19b) and (2.19a). Similarly, we have

$$
\begin{gathered}
q x^{-3} g\left(-q^{3} x^{2}, q^{4}\right)=q^{-2} x^{-5} m\left(-q^{-1} x^{6}, q^{12}, q^{6} x^{4}\right)-q^{-5} x^{-7} m\left(-q^{-5} x^{-6}, q^{12}, q^{6} x^{4}\right) \\
=q^{-2} x^{-5} m\left(-q^{-1} x^{6}, q^{12}, q^{6} x^{4}\right)+x^{-1}-x^{-1} m\left(-q^{7} x^{-6}, q^{12}, q^{6} x^{4}\right),
\end{gathered}
$$

where the last line follows from (2.19e). Combining (2.28), (2.29), and (2.30) and then simplifying yields

$$
\begin{aligned}
g(x, q)= & -x^{-1}+q x^{-3} g\left(-q x^{-2}, q^{4}\right)-q g\left(-q x^{2}, q^{4}\right) \\
& +\frac{x^{-1} J_{6}^{3} \bar{J}_{1,6} J_{6,12}}{j\left(q^{6} x^{4} ; q^{12}\right) j\left(x^{2} ; q^{6}\right)} \cdot\left[\frac{1}{j\left(q x ; q^{3}\right) j\left(-q^{5} x^{2} ; q^{6}\right)}+\frac{x}{j\left(q^{2} x ; q^{3}\right) j\left(-q x^{2} ; q^{6}\right)}\right] \\
= & -x^{-1}+q x^{-3} g\left(-q x^{-2}, q^{4}\right)-q g\left(-q x^{2}, q^{4}\right) \\
& +\frac{x^{-1} J_{6}^{3} \bar{J}_{1,6} J_{6,12}}{j\left(q^{6} x^{4} ; q^{12}\right) j\left(x^{2} ; q^{6}\right)} \cdot \frac{j\left(-x ; q^{3}\right) j\left(q^{3} x^{2} ; q^{6}\right)}{j\left(q x ; q^{3}\right) j\left(-q^{5} x^{2} ; q^{6}\right) j\left(q^{2} x ; q^{3}\right) j\left(-q x^{2} ; q^{6}\right)} \cdot \frac{J_{2,12}^{2} J_{4,12}}{J_{12}^{3}},
\end{aligned}
$$

where the last equality follows from Proposition 2.5. The result then follows from elementary theta function properties.

2.3. Properties of Hecke-type double sums. We recall from [10] some useful Hecketype double sum identities:

Proposition 2.16. For $x, y \in \mathbb{C}^{*}$

$$
\begin{aligned}
f_{a, b, c}(x, y, q)= & f_{a, b, c}\left(-x^{2} q^{a},-y^{2} q^{c}, q^{4}\right)-x f_{a, b, c}\left(-x^{2} q^{3 a},-y^{2} q^{c+2 b}, q^{4}\right) \\
& -y f_{a, b, c}\left(-x^{2} q^{a+2 b},-y^{2} q^{3 c}, q^{4}\right)+x y q^{b} f_{a, b, c}\left(-x^{2} q^{3 a+2 b},-y^{2} q^{3 c+2 b}, q^{4}\right), \\
f_{a, b, c}(x, y, q)= & -\frac{q^{a+b+c}}{x y} f_{a, b, c}\left(q^{2 a+b} / x, q^{2 c+b} / y, q\right),
\end{aligned}
$$




$$
\begin{aligned}
& f_{a, b, c}(x, y, q)=-y f_{a, b, c}\left(q^{b} x, q^{c} y, q\right)+j\left(x ; q^{a}\right), \\
& f_{a, b, c}(x, y, q)=-x f_{a, b, c}\left(q^{a} x, q^{b} y, q\right)+j\left(y ; q^{c}\right) .
\end{aligned}
$$

We state and prove a corollary to Theorem 1.3 .

Corollary 2.17. For generic $x, y \in \mathbb{C}^{*}$

$$
f_{3,5,3}(x, y, q)=g_{3,5,3}(x, y, q,-1,-1)+\theta_{3,2}(x, y, q)
$$

where

$$
\begin{aligned}
g_{3,5,3}(x, y, q,-1,-1):= & \left.\sum_{t=0}^{2}(-y)^{t} q^{3} \begin{array}{c}
t \\
2
\end{array}\right) j\left(q^{5 t} x ; q^{3}\right) m\left(-q^{27-16 t} \cdot \frac{y^{3}}{x^{5}}, q^{48},-1\right) \\
& +\sum_{t=0}^{2}(-x)^{t} q^{3\left(\begin{array}{c}
t \\
2
\end{array}\right)} j\left(q^{5 t} y ; q^{3}\right) m\left(-q^{27-16 t} \cdot \frac{x^{3}}{y^{5}}, q^{48},-1\right),
\end{aligned}
$$

and

$$
\begin{aligned}
\theta_{3,2}(x, y, q):= & \frac{1}{\bar{J}_{0,48}} \cdot \frac{x^{1 / 2} y^{1 / 2} q^{-11 / 2} j\left(q^{5} x y ; q^{8}\right) \bar{J}_{8,32}}{2 j\left(q^{5} y^{5} / x^{3} ; q^{16}\right) j\left(q^{5} x^{5} / y^{3} ; q^{16}\right)} \\
& \cdot\left[j\left(-q^{5 / 2} x^{5 / 2} / y^{3 / 2} ; q^{8}\right) j\left(-q^{5 / 2} y^{5 / 2} / x^{3 / 2} ; q^{8}\right) j\left(q^{3 / 2} y^{3 / 2} / x^{3 / 2} ; q^{3}\right)\right. \\
& \left.\quad-j\left(q^{5 / 2} x^{5 / 2} / y^{3 / 2} ; q^{8}\right) j\left(q^{5 / 2} y^{5 / 2} / x^{3 / 2} ; q^{8}\right) j\left(-q^{3 / 2} y^{3 / 2} / x^{3 / 2} ; q^{3}\right)\right] .
\end{aligned}
$$

Proof of Corollary 2.17. Identity (2.35) easily follows from the definition (1.4). For (2.36) we first substitute $n=3$ and $p=2$ into the definition of $\theta_{3,2}(x, y, q)$ to obtain

$$
\begin{gathered}
\theta_{3,2}(x, y, q)=\left[-\frac{y^{2} j\left(-x^{3} / y^{3} ; q^{12}\right) j\left(q^{10} x^{2} y^{2} ; q^{32}\right)}{q^{4} x j\left(q^{5} y^{5} / x^{3}, q^{5} x^{5} / y^{3} ; q^{32}\right)}+\frac{y^{3} j\left(-q^{6} x^{3} / y^{3} ; q^{12}\right) j\left(q^{26} x^{2} y^{2} ; q^{32}\right)}{q^{3} x j\left(q^{5} y^{5} / x^{3}, q^{21} x^{5} / y^{3} ; q^{32}\right)}\right. \\
\left.+\frac{q^{3} y^{2} j\left(-q^{-6} x^{3} / y^{3} ; q^{12}\right) j\left(q^{26} x^{2} y^{2} ; q^{32}\right)}{j\left(q^{21} y^{5} / x^{3}, q^{5} x^{5} / y^{3} ; q^{32}\right)}-\frac{q^{9} y^{3} j\left(-x^{3} / y^{3} ; q^{12}\right) j\left(q^{42} x^{2} y^{2} ; q^{32}\right)}{j\left(q^{21} y^{5} / x^{3}, q^{21} x^{5} / y^{3} ; q^{32}\right)}\right] \cdot \frac{J_{32}^{3}}{\bar{J}_{0,48}} .
\end{gathered}
$$

We then combine the first and fourth summands as well as the second and third summands using (2.3b). For example, we first use (2.1a) to write

$$
j\left(q^{42} x^{2} y^{2} ; q^{32}\right)=-x^{-2} y^{-2} q^{-10} j\left(q^{10} x^{2} y^{2} ; q^{32}\right) .
$$

This allows us to rewrite the sum of the first and fourth summands as

$$
\begin{aligned}
& -\frac{y j\left(-x^{3} / y^{3} ; q^{12}\right) j\left(q^{10} x^{2} y^{2} ; q^{32}\right)}{q x^{2} j\left(q^{5} y^{5} / x^{3}, q^{5} x^{5} / y^{3}, q^{21} y^{5} / x^{3}, q^{21} x^{5} / y^{3} ; q^{32}\right)} \\
& \cdot\left[j\left(q^{5} y^{5} / x^{3}, q^{5} x^{5} / y^{3} ; q^{32}\right)-x y q^{-3} j\left(q^{21} y^{5} / x^{3}, q^{21} x^{5} / y^{3} ; q^{32}\right)\right] .
\end{aligned}
$$

We evaluate the bracketed expression by substituting $q \rightarrow q^{16}, x \rightarrow y q^{-3}, y \rightarrow-q^{8} y^{4} / x^{4}$, in (2.3b), and we rewrite the denominator by using (2.1d). Using (2.1a) to have

$$
j\left(x y q^{-3} ; q^{16}\right)=-q^{-16} q^{-3} x y j\left(q^{13} x y ; q^{16}\right)=-q^{-3} x y j\left(q^{13} x y ; q^{16}\right),
$$


we see that the sum of the first and fourth summands is

$$
-\frac{y^{2} j\left(-x^{3} / y^{3} ; q^{12}\right) j\left(q^{10} x^{2} y^{2} ; q^{32}\right) j\left(q^{13} x y ; q^{16}\right) j\left(-q^{8} y^{4} / x^{4} ; q^{16}\right) J_{16}^{2}}{q^{4} x j\left(q^{5} y^{5} / x^{3} ; q^{16}\right) j\left(q^{5} x^{5} / y^{3} ; q^{16}\right) J_{32}^{4}} .
$$

The second and third summands can be combined in a similar way, and it follows that we can write

$$
\begin{aligned}
\theta_{3,2}(x, y, q)= & \frac{1}{\bar{J}_{0,48}} \cdot \frac{x^{3} y^{-1} q^{-3} J_{16,32}}{j\left(q^{5} y^{5} / x^{3} ; q^{16}\right) j\left(q^{5} x^{5} / y^{3} ; q^{16}\right)} \\
& \cdot\left[j\left(-q^{6} x^{3} / y^{3} ; q^{12}\right) j\left(q^{26} x^{2} y^{2} ; q^{32}\right) j\left(-y^{4} / x^{4} ; q^{16}\right) j\left(q^{5} y x ; q^{16}\right)\right. \\
& \left.\quad-x^{-4} y^{3} q^{-1} j\left(-x^{3} / y^{3} ; q^{12}\right) j\left(q^{10} x^{2} y^{2} ; q^{32}\right) j\left(-q^{8} y^{4} / x^{4} ; q^{16}\right) j\left(q^{13} y x ; q^{16}\right)\right] .
\end{aligned}
$$

Using (2.1g) and (2.1d) we obtain

$$
\begin{aligned}
\theta_{3,2}(x, y, q)= & \frac{1}{\bar{J}_{0,48}} \cdot \frac{x^{3} y^{-1} q^{-3}}{j\left(q^{5} y^{5} / x^{3} ; q^{16}\right) j\left(q^{5} x^{5} / y^{3} ; q^{16}\right)} \\
& \cdot\left[j\left(-q^{6} x^{3} / y^{3} ; q^{12}\right) j\left(-q^{13} x y, q^{13} x y,-y^{4} / x^{4}, q^{5} y x ; q^{16}\right)\right. \\
& \left.\quad-x^{-4} y^{3} q^{-1} j\left(-x^{3} / y^{3} ; q^{12}\right) j\left(-q^{5} x y, q^{5} x y,-q^{8} y^{4} / x^{4}, q^{13} y x ; q^{16}\right)\right] \\
= & \frac{1}{\bar{J}_{0,48}} \cdot \frac{x^{3} y^{-1} q^{-3} j\left(q^{5} x y ; q^{8}\right) \bar{J}_{8,32}}{j\left(q^{5} y^{5} / x^{3} ; q^{16}\right) j\left(q^{5} x^{5} / y^{3} ; q^{16}\right)} \\
& \cdot\left[j\left(-q^{6} x^{3} / y^{3} ; q^{12}\right) j\left(-q^{13} x y ; q^{16}\right) j\left(-y^{4} / x^{4} ; q^{16}\right)\right. \\
& \left.\quad-x^{-4} y^{3} q^{-1} j\left(-x^{3} / y^{3} ; q^{12}\right) j\left(-q^{5} x y ; q^{16}\right) j\left(-q^{8} y^{4} / x^{4} ; q^{16}\right)\right] .
\end{aligned}
$$

From (2.3c) and (2.3d), we have

$$
\begin{aligned}
j\left(q^{5 / 2} x^{5 / 2} / y^{3 / 2}, q^{5 / 2} y^{5 / 2} / x^{3 / 2} ; q^{8}\right)- & j\left(-q^{5 / 2} x^{5 / 2} / y^{3 / 2},-q^{5 / 2} y^{5 / 2} / x^{3 / 2} ; q^{8}\right) \\
& =-2 q^{5 / 2} x^{5 / 2} / y^{3 / 2} j\left(-y^{4} / x^{4},-q^{13} x y ; q^{16}\right), \\
j\left(q^{5 / 2} x^{5 / 2} / y^{3 / 2}, q^{5 / 2} y^{5 / 2} / x^{3 / 2} ; q^{8}\right)+ & j\left(-q^{5 / 2} x^{5 / 2} / y^{3 / 2},-q^{5 / 2} y^{5 / 2} / x^{3 / 2} ; q^{8}\right) \\
& =2 j\left(-q^{8} y^{4} / x^{4},-q^{5} x y ; q^{16}\right) .
\end{aligned}
$$

Substituting (2.39) and (2.40) into (2.38) and collecting terms yields

$$
\begin{aligned}
& \theta_{3,2}(x, y, q)=\frac{1}{\bar{J}_{0,48}} \cdot \frac{x^{1 / 2} y^{1 / 2} q^{-11 / 2} j\left(q^{5} x y ; q^{8}\right) \bar{J}_{8,32}}{2 j\left(q^{5} y^{5} / x^{3} ; q^{16}\right) j\left(q^{5} x^{5} / y^{3} ; q^{16}\right)} \\
& \quad \cdot\left[j\left(-q^{5 / 2} x^{5 / 2} / y^{3 / 2},-q^{5 / 2} y^{5 / 2} / x^{3 / 2} ; q^{8}\right)\left(j\left(-q^{6} x^{3} / y^{3} ; q^{12}\right)-\frac{q^{3 / 2} y^{3 / 2}}{x^{3 / 2}} j\left(-x^{3} / y^{3} ; q^{12}\right)\right)\right. \\
& \left.\quad-j\left(q^{5 / 2} x^{5 / 2} / y^{3 / 2}, q^{5 / 2} y^{5 / 2} / x^{3 / 2} ; q^{8}\right)\left(j\left(-q^{6} x^{3} / y^{3} ; q^{12}\right)+\frac{q^{3 / 2} y^{3 / 2}}{x^{3 / 2}} j\left(-x^{3} / y^{3} ; q^{12}\right)\right)\right] .
\end{aligned}
$$


Using (2.1f) with $m=2$ gives the desired result.

2.4. Third order mock theta functions in terms of the Appell-Lerch sums. We finish the preliminaries section by recalling the following identities from [10] which were shown using results of Watson [15] and Appell-Lerch sum properties.

$$
\begin{aligned}
\psi(q): & =\sum_{n \geq 1} \frac{q^{n^{2}}}{\left(q ; q^{2}\right)_{n}}=q g\left(q, q^{4}\right)=-q^{-1} m\left(q, q^{12}, q^{2}\right)-m\left(q^{5}, q^{12}, q^{2}\right) \\
& =-m\left(q,-q^{3},-q\right)+\frac{q J_{12}^{3}}{J_{4} J_{3,12}} \\
\nu(q): & =\sum_{n \geq 0} \frac{q^{n(n+1)}}{\left(-q ; q^{2}\right)_{n+1}}=g(i \sqrt{q}, q)=q^{-1} m\left(q^{2}, q^{12},-q^{3}\right)+q^{-1} m\left(q^{2}, q^{12},-q^{9}\right) \\
& =2 q^{-1} m\left(q^{2}, q^{12},-q^{3}\right)+\frac{J_{1} J_{3,12}}{J_{2}} \\
\phi(q): & =\sum_{n \geq 0} \frac{q^{n^{2}}}{\left(-q^{2} ; q^{2}\right)_{n}}=(1-i)(1+i g(i, q)) \\
& =m\left(q^{5}, q^{12}, q^{4}\right)+m\left(q^{5}, q^{12}, q^{8}\right)+q^{-1} m\left(q, q^{12}, q^{4}\right)+q^{-1} m\left(q, q^{12}, q^{8}\right) \\
& =2 m\left(q,-q^{3},-1\right)+\frac{2 q J_{12}^{3}}{J_{4} J_{3,12}}
\end{aligned}
$$

\section{Proof of Theorem 1.5}

We prove identity (1.5). Focusing on the right hand side, we have

$$
\begin{aligned}
\frac{1}{(q)_{\infty}} & \sum_{n=0}^{\infty}(-1)^{n} q^{2 n^{2}+n}\left(1+q^{2 n+1}\right) \sum_{j=-n}^{n} q^{-\left(\begin{array}{c}
j+1 \\
2
\end{array}\right)} \\
& =\frac{1}{(q)_{\infty}}\left(\sum_{n=0}^{\infty}(-1)^{n} q^{2 n^{2}+n} \sum_{j=-n}^{n} q^{-\left(\begin{array}{c}
j+1 \\
2
\end{array}\right)}+\sum_{n=0}^{\infty}(-1)^{n} q^{2 n^{2}+3 n+1} \sum_{j=-n}^{n} q^{-\left(\begin{array}{c}
j+1 \\
2
\end{array}\right)}\right) \\
& =\frac{1}{(q)_{\infty}}\left(\sum_{\substack{n+j>0 \\
n-j \geq 0}}-\sum_{\substack{n+j<0 \\
n-j<0}}\right)(-1)^{n} q^{2 n^{2}+n-\left(\begin{array}{c}
j+1 \\
2
\end{array}\right)},
\end{aligned}
$$

where in the last line we replaced $n$ with $-n-1$. With a few more straightforward operations, we have

$$
\frac{1}{(q)_{\infty}}\left(\sum_{\substack{n+j \geq 0 \\
n-j \geq 0}}-\sum_{\substack{n+j<0 \\
n-j<0}}\right)(-1)^{n} q^{2 n^{2}+n-\left(\begin{array}{c}
j+1 \\
2
\end{array}\right)}
$$




$$
\begin{aligned}
& =\frac{1}{(q)_{\infty}}\left(\sum_{\substack{r \equiv s, s \geq 0 \\
(\bmod 2)}}-\sum_{\substack{r, s<0 \\
r \equiv s}}\right)(-1)^{\frac{r+s}{2}} q^{\frac{3}{8} r^{2}+\frac{5}{4} r s+\frac{3}{8} s^{2}+\frac{1}{4} r+\frac{3}{4} s} \\
& =\frac{1}{(q)_{\infty}}\left(f_{3,5,3}\left(q^{2}, q^{3}, q\right)-q^{3} f_{3,5,3}\left(q^{6}, q^{7}, q\right)\right) \\
& =\frac{1}{(q)_{\infty}}\left(2 f_{3,5,3}\left(q^{2}, q^{3}, q\right)-(q)_{\infty}\right),
\end{aligned}
$$

where the first equality follows from the substitutions $r=n+j$ and $s=n-j$, the second equality follows from considering the cases $r, s$ even and $r, s$ odd. So to prove (1.5), it suffices to show

$$
J_{1} \cdot(1+\psi(q))=f_{3,5,3}\left(q^{2}, q^{3}, q\right) .
$$

We first compute $g_{3,5,3}\left(q^{2}, q^{3}, q,-1,-1\right)$. Using Corollary 2.17, we have

$$
\begin{aligned}
g_{3,5,3}\left(q^{2}, q^{3}, q,-1,-1\right)= & j\left(q^{2} ; q^{3}\right) m\left(-q^{26}, q^{48},-1\right)+j\left(q^{3} ; q^{3}\right) m\left(-q^{18}, q^{48},-1\right) \\
& -q^{3} j\left(q^{7} ; q^{3}\right) m\left(-q^{10}, q^{48},-1\right)-q^{2} j\left(q^{8} ; q^{3}\right) m\left(-q^{2}, q^{48},-1\right) \\
& +q^{9} j\left(q^{12} ; q^{3}\right) m\left(-q^{-6}, q^{48},-1\right)+q^{7} j\left(q^{13} ; q^{3}\right) m\left(-q^{-14}, q^{48},-1\right) \\
= & J_{1} m\left(-q^{26}, q^{48},-1\right)-q^{-2} J_{1} m\left(-q^{10}, q^{48},-1\right) \\
& -q^{-5} J_{1} m\left(-q^{2}, q^{48},-1\right)+q^{-15} J_{1} m\left(-q^{-14}, q^{48},-1\right),
\end{aligned}
$$

by (2.1a). Using (2.19c) and (2.19b),

$$
\begin{aligned}
g_{3,5,3}\left(q^{2}, q^{3}, q,-1,-1\right)= & J_{1}+J_{1} q^{-22} m\left(-q^{-22}, q^{48},-1\right)+q^{-12} J_{1} m\left(-q^{-10}, q^{48},-1\right) \\
& +q^{-7} J_{1} m\left(-q^{-2}, q^{48},-1\right)-q^{-1} J_{1} m\left(-q^{14}, q^{48},-1\right) \\
= & J_{1}-J_{1} m\left(-q^{22}, q^{48},-1\right)+q^{-12} J_{1} m\left(-q^{-10}, q^{48},-1\right) \\
& +q^{-7} J_{1} m\left(-q^{-2}, q^{48},-1\right)-q^{-1} J_{1} m\left(-q^{14}, q^{48},-1\right) .
\end{aligned}
$$

Using Corollary 2.11, we have

$$
\begin{aligned}
m\left(q, q^{12}, q^{2}\right)= & m\left(-q^{14}, q^{48},-1\right)-q^{-11} m\left(-q^{-10}, q^{48},-1\right) \\
& -\frac{J_{24}^{3}}{J_{3,12} J_{14,24} \bar{J}_{0,48}} \cdot\left[\frac{J_{16,24} \bar{J}_{4,48}}{J_{2,24}}-q^{3} \cdot \frac{J_{28,24} \bar{J}_{28,48}}{J_{14,24}}\right] \\
= & m\left(-q^{14}, q^{48},-1\right)-q^{-11} m\left(-q^{-10}, q^{48},-1\right) \\
& -\frac{J_{24}^{3}}{J_{3,12} J_{14,24} \bar{J}_{0,48}} \cdot\left[\frac{J_{16,24} \bar{J}_{4,48}}{J_{2,24}}+q^{-1} \cdot \frac{J_{4,24} \bar{J}_{28,48}}{J_{14,24}}\right],
\end{aligned}
$$

and

$$
\begin{gathered}
m\left(q^{5}, q^{12}, q^{2}\right)=m\left(-q^{22}, q^{48},-1\right)-q^{-7} m\left(-q^{-2}, q^{48},-1\right) \\
-\frac{J_{24}^{3}}{J_{7,12} J_{22,24} \bar{J}_{0,48}} \cdot\left[\frac{J_{24,24} \bar{J}_{4,48}}{J_{2,24}}-q^{7} \cdot \frac{J_{36,24} \bar{J}_{28,48}}{J_{14,24}}\right]
\end{gathered}
$$


ON THREE THIRD ORDER MOCK THETA FUNCTIONS AND HECKE-TYPE DOUBLE SUMS

$$
=m\left(-q^{22}, q^{48},-1\right)-q^{-7} m\left(-q^{-2}, q^{48},-1\right)-q^{-5} \cdot \frac{J_{24}^{3} J_{12,24} \bar{J}_{28,48}}{J_{7,12} J_{22,24} \bar{J}_{0,48} J_{14,24}} .
$$

Substituting (3.3) and (3.4) into (3.2) and using (2.42), we have

$$
\begin{aligned}
g_{3,5,3}\left(q^{2}, q^{3}, q,-1,-1\right)= & J_{1}(1+\psi(q))-q^{-5} \cdot \frac{J_{24}^{3} J_{1} J_{12,24} \bar{J}_{28,48}}{J_{7,12} J_{22,24} \bar{J}_{0,48} J_{14,24}} \\
& -q^{-1} \cdot \frac{J_{1} J_{24}^{3}}{J_{3,12} J_{14,24} \bar{J}_{0,48}}\left[\frac{J_{16,24} \bar{J}_{4,48}}{J_{2,24}}+q^{-1} \cdot \frac{J_{4,24} \bar{J}_{28,48}}{J_{14,24}}\right] \\
= & J_{1}(1+\psi(q))-q^{-1} \cdot \frac{J_{24}^{3} J_{8,24} \bar{J}_{4,48} J_{1}}{J_{3,12} J_{10,24} \bar{J}_{0,48} J_{2,24}} \\
& -q^{-5} \cdot \frac{J_{24}^{3} \bar{J}_{28,48} J_{1}}{\bar{J}_{0,48} J_{10,24}}\left[\frac{J_{12,24}}{J_{7,12} J_{22,24}}+q^{3} \cdot \frac{J_{4,24}}{J_{3,12} J_{14,24}}\right],
\end{aligned}
$$

where in the last equality we grouped the first and third summands. Using Proposition 2.1 with $q \rightarrow q^{24}, a=q^{13}, b=q^{9}, c=q^{6}, d=q$, to evaluate the bracketed expression we obtain

$$
\begin{aligned}
g_{3,5,3} & \left.q^{2}, q^{3}, q,-1,-1\right)=J_{1}(1+\psi(q))-q^{-1} \cdot \frac{J_{24}^{3} J_{8,24} \bar{J}_{4,48} J_{1}}{J_{3,12} J_{10,24} \bar{J}_{0,48} J_{2,24}} \\
& -q^{-5} \cdot \frac{J_{24}^{3} \bar{J}_{28,48} J_{1}}{\bar{J}_{0,48} J_{10,24}} \cdot \frac{J_{14,24} J_{8,24} J_{19,24} J_{17,24}}{J_{3,12} J_{7,12} J_{2,24} J_{10,24}} \cdot \frac{J_{12}}{J_{24}^{2}} \\
= & \left.J_{1}(1+\psi(q))-q^{-1} \cdot \frac{J_{1} J_{24} J_{8} \bar{J}_{4,48} J_{12}}{J_{3,12} J_{2,12} \bar{J}_{0,48}}-q^{-5} \cdot \frac{J_{1} J_{24} J_{8} J_{12} \bar{J}_{28,48}}{\bar{J}_{0,48} J_{2,12} J_{3,12}} \quad(\text { by (2.1d) })\right) \\
= & J_{1}(1+\psi(q))-q^{-5} \cdot \frac{J_{1} J_{24} J_{8} J_{12}}{J_{3,12} J_{2,12} \bar{J}_{0,48}}\left[\bar{J}_{20,48}+q^{4} \bar{J}_{44,48}\right] .
\end{aligned}
$$

Using (2.1f) with $m=2$ yields

$$
g_{3,5,3}\left(q^{2}, q^{3}, q,-1,-1\right)=J_{1}(1+\psi(q))-q^{-5} \cdot \frac{J_{1} J_{24} J_{8} J_{12} \bar{J}_{4,12}}{\bar{J}_{0,48} J_{2,12} J_{3,12}} .
$$

We compute $\theta_{3,2}\left(q^{2}, q^{3}, q\right)$. Using Corollary 2.17, we have

$$
\begin{aligned}
\theta_{3,2}\left(q^{2}, q^{3}, q\right) & =\frac{1}{2 \bar{J}_{0,48}} \cdot \frac{q^{-3} j\left(q^{10} ; q^{8}\right)}{j\left(q^{14} ; q^{16}\right) j\left(q^{6} ; q^{16}\right)} \cdot \frac{J_{16}^{2}}{J_{8}} \cdot\left[0-j\left(q^{3} ; q^{8}\right) j\left(q^{7} ; q^{8}\right) j\left(-q^{3} ; q^{3}\right)\right] \\
& =\frac{1}{2 \bar{J}_{0,48}} \cdot \frac{q^{-5} J_{2,8}}{J_{2,16} J_{6,16}} \cdot \frac{J_{16}^{2}}{J_{8}} \cdot J_{3,8} J_{7,8} \bar{J}_{0,3} .
\end{aligned}
$$

Simplying with elementary theta function properties shows that (3.6) is equal to the quotient of theta functions in (3.5), and the result follows. 
We prove identity (1.6). Focusing on the right hand side and replacing $q$ with $q^{2}$, we have

$$
\frac{1}{\left(q^{2} ; q^{2}\right)_{\infty}} \sum_{n=0}^{\infty}(-1)^{n} q^{4 n^{2}+4 n} \sum_{j=-n}^{n} q^{-j^{2}-j}=\frac{1}{2 \cdot\left(q^{2} ; q^{2}\right)_{\infty}}\left(\sum_{\substack{n+j>0 \\ n-j \geq 0}}-\sum_{\substack{n+j<0 \\ n-j<0}}\right)(-1)^{n} q^{4 n^{2}+4 n-j^{2}-j}
$$

The quadratic part of the exponent in the Hecke-type sum factors. Letting $j=2 n-k$, we can rewrite (3.7) in terms of $k$ and $n$. For fixed $k$, the sum over $n$ is a finite geometric series. So (3.7) is equivalent to

$$
\frac{1}{2 \cdot\left(q^{2} ; q^{2}\right)_{\infty}} \sum_{k=0}^{\infty} q^{-k^{2}+k} \cdot \frac{\left(-q^{4 k+2}\right)^{\lceil k / 3\rceil}-\left(-q^{4 k+2}\right)^{k+1}}{1+q^{4 k+2}}
$$

where $\lceil\cdot\rceil$ is the ceiling function. By standard series manipulations, we can rewrite (3.8) as a sum of terms of the form $j * m$ :

$$
\begin{aligned}
\frac{1}{2 \cdot\left(q^{2} ; q^{2}\right)_{\infty}} & \cdot\left(\bar{J}_{22,24} m\left(q^{4}, q^{24},-q^{22}\right)-q^{-2} \bar{J}_{10,24} m\left(q^{4}, q^{24},-q^{10}\right)-\bar{J}_{6,24} m\left(q^{12}, q^{24},-q^{6}\right)\right. \\
& +\bar{J}_{6,24} m\left(q^{12}, q^{24},-q^{18}\right)-\bar{J}_{14,24} q^{-2} m\left(q^{4}, q^{24},-q^{10}\right)+\bar{J}_{2,24} m\left(q^{4}, q^{24},-q^{22}\right) \\
& +\bar{J}_{2,24} m\left(q^{4}, q^{24},-q^{2}\right)-\bar{J}_{10,24} q^{-2} m\left(q^{4}, q^{24},-q^{14}\right)+\bar{J}_{6,24} m\left(q^{12}, q^{24},-q^{6}\right) \\
& \left.-\bar{J}_{14,24} q^{-2} m\left(q^{4}, q^{24},-q^{14}\right)+\bar{J}_{2,24} m\left(q^{4}, q^{24},-q^{2}\right)-\bar{J}_{6,24} m\left(q^{12}, q^{24},-q^{-6}\right)\right) .
\end{aligned}
$$

This reduces to

$$
\begin{aligned}
& \frac{1}{J_{2}} \cdot\left(\bar{J}_{22,24} m\left(q^{4}, q^{24},-q^{22}\right)+\bar{J}_{2,24} m\left(q^{4}, q^{24},-q^{2}\right)-\bar{J}_{10,24} q^{-2} m\left(q^{4}, q^{24},-q^{10}\right)\right. \\
& \left.\quad-\bar{J}_{14,24} q^{-2} m\left(q^{4}, q^{24},-q^{14}\right)\right) .
\end{aligned}
$$

Using identity (2.19f), we have

$$
\begin{aligned}
& m\left(q^{4}, q^{24},-q^{22}\right)=m\left(q^{4}, q^{24}, q^{6}\right)+\frac{J_{24}^{3} \bar{J}_{16,24} \bar{J}_{8,24}}{\bar{J}_{2,24}^{2} J_{6,24} J_{10,24}} \\
& m\left(q^{4}, q^{24},-q^{2}\right)=m\left(q^{4}, q^{24}, q^{6}\right)+\frac{q^{2} J_{24}^{3} \bar{J}_{4,24} \bar{J}_{12,24}}{\bar{J}_{2,24} \bar{J}_{6,24} J_{6,24} J_{10,24}} \\
& m\left(q^{4}, q^{24},-q^{10}\right)=m\left(q^{4}, q^{24}, q^{6}\right)+\frac{q^{6} J_{24}^{3} \bar{J}_{4,24} \bar{J}_{20,24}}{\bar{J}_{10,24}^{2} J_{6,24} J_{10,24}} \\
& m\left(q^{4}, q^{24},-q^{14}\right)=m\left(q^{4}, q^{24}, q^{6}\right)+\frac{q^{6} J_{24}^{3} \bar{J}_{8,24} \bar{J}_{0,24}}{\bar{J}_{14,24} \bar{J}_{18,24} J_{6,24} J_{10,24}}
\end{aligned}
$$


Using (2.1f), we have $J_{2}=\bar{J}_{10,24}-q^{2} \bar{J}_{22,24}$. Thus (3.9) is equivalent to

$$
\begin{aligned}
-2 q^{-2} m\left(q^{4}, q^{24}, q^{6}\right) & +\frac{J_{24}^{3}}{J_{2} J_{6,24} J_{10,24}}\left[\frac{\bar{J}_{8,24}^{2}}{\bar{J}_{2,24}}-\frac{q^{4} \bar{J}_{4,24}^{2}}{\bar{J}_{10,24}}\right] \\
& +\frac{q^{2} J_{24}^{3}}{J_{2} J_{6,24} J_{10,24}}\left[\frac{\bar{J}_{4,24} \bar{J}_{12,24}}{\bar{J}_{6,24}}-\frac{q^{2} \bar{J}_{8,24} \bar{J}_{0,24}}{\bar{J}_{6,24}}\right] .
\end{aligned}
$$

Focusing on the second summand of (3.10), we find that

$$
\begin{aligned}
\frac{J_{24}^{3}}{J_{2} J_{6,24} J_{10,24}}\left[\frac{\bar{J}_{8,24}^{2}}{\bar{J}_{2,24}}-\frac{q^{4} \bar{J}_{4,24}^{2}}{\bar{J}_{10,24}}\right] & =\frac{J_{24}^{3}}{J_{2} J_{6,24} J_{10,24}} \cdot \frac{1}{\bar{J}_{2,24} \bar{J}_{14,24}}\left[\bar{J}_{8,24}^{2} \bar{J}_{10,24}-q^{4} \bar{J}_{4,24}^{2} \bar{J}_{2,24}\right] \\
& =\frac{J_{24}^{3}}{J_{2} J_{6,24} J_{10,24}} \cdot \frac{1}{\bar{J}_{2,24} \bar{J}_{14,24}} \cdot \frac{J_{10,24}^{2} J_{12,24} J_{4,24}}{\bar{J}_{6,24}} \\
& =\frac{J_{24}^{3}}{J_{2} J_{6,24} J_{10,24}} \cdot \frac{J_{10,24}^{2} J_{12} J_{2,12}}{\bar{J}_{6,24} J_{24}^{2}}
\end{aligned}
$$

where the second equality follows from Proposition 2.1 with $q \rightarrow q^{24}, a=q^{12}, b=q^{2}$, $c=-q^{4}, d=-q^{8}$, and the last equality follows from elementary theta function properties. Focusing on the third summand of (3.10), we obtain

$$
\begin{aligned}
\frac{q^{2} J_{24}^{3}}{J_{2} J_{6,24} J_{10,24}}\left[\frac{\bar{J}_{4,24} \bar{J}_{12,24}}{\bar{J}_{6,24}}-\frac{q^{2} \bar{J}_{8,24} \bar{J}_{0,24}}{\bar{J}_{6,24}}\right] & =\frac{q^{2} J_{24}^{3}}{J_{2} J_{6,24} J_{10,24} \bar{J}_{6,24}} \cdot\left[\bar{J}_{4,24} \bar{J}_{12,24}-q^{2} \bar{J}_{8,24} \bar{J}_{0,24}\right] \\
& =\frac{q^{2} J_{24}^{3} J_{2,12}^{2}}{J_{2} J_{6,24} J_{10,24} \bar{J}_{6,24}}
\end{aligned}
$$

where the last line follows from (2.3b) with $q \rightarrow q^{12}, x=q^{2}, y=q^{2}$. Assembling the pieces shows that (3.7) is equivalent to

$$
\begin{aligned}
& -2 q^{-2} m\left(q^{4}, q^{24}, q^{6}\right)+\frac{J_{24}^{3} J_{2,12}}{J_{2} J_{6,24} J_{10,24} \bar{J}_{6,24}} \cdot \frac{J_{12}}{J_{24}^{2}} \cdot\left[q^{2} J_{2,12} \frac{J_{24}^{2}}{J_{12}}+J_{10,24}^{2}\right] \\
& =-2 q^{-2} m\left(q^{4}, q^{24}, q^{6}\right)+\frac{J_{24}^{3} J_{2,12}}{J_{2} J_{6,24} J_{10,24} \bar{J}_{6,24}} \cdot \frac{J_{12}}{J_{24}^{2}} \cdot\left[q^{2} J_{2,24} J_{14,24}+J_{10,24}^{2}\right] \\
& =-2 q^{-2} m\left(q^{4}, q^{24}, q^{6}\right)+\frac{J_{24}^{3} J_{2,12}}{J_{2} J_{6,24} J_{10,24} \bar{J}_{6,24}} \cdot \frac{J_{12}}{J_{24}^{2}} \cdot J_{10,24} \cdot\left[q^{2} J_{2,24}+J_{10,24}\right] \\
& =-2 q^{-2} m\left(q^{4}, q^{24}, q^{6}\right)+\frac{J_{24}^{3} J_{2,12}}{J_{2} J_{6,24} J_{10,24} \bar{J}_{6,24}} \cdot \frac{J_{12}}{J_{24}^{2}} \cdot J_{10,24} \cdot j\left(-q^{2} ;-q^{6}\right) .
\end{aligned}
$$

Elementary theta function properties shows that this is $\nu\left(-q^{2}\right)$. 


\section{Proof of Theorem 1.6}

We prove identity (1.11). Focusing on the left hand side, we have

$$
\begin{aligned}
\sum_{n=0}^{\infty} \frac{q^{2 n^{2}}}{(-q ; q)_{2 n}} & =\frac{1}{\left(q^{2} ; q^{2}\right)_{\infty}} \sum_{n=0}^{\infty} q^{4 n^{2}+n}\left(1-q^{6 n+3}\right) \sum_{j=-n}^{n}(-1)^{j} q^{-j^{2}} \\
& =\frac{1}{\left(q^{2} ; q^{2}\right)_{\infty}}\left(\sum_{\substack{n+j \geq 0 \\
n-j \geq 0}}-\sum_{\substack{n+j<0 \\
n-j<0}}\right)(-1)^{j} q^{4 n^{2}+n-j^{2}} \\
& =\frac{1}{\left(q^{2} ; q^{2}\right)_{\infty}}\left(f_{3,5,3}\left(q^{4}, q^{4}, q^{2}\right)+q^{5} f_{3,5,3}\left(q^{12}, q^{12}, q^{2}\right)\right) \\
& =\frac{1}{\left(q^{2} ; q^{2}\right)_{\infty}}\left(f_{3,5,3}\left(q^{5 / 4},-q^{5 / 4},-q^{1 / 2}\right)\right) .
\end{aligned}
$$

We first compute $g_{3,5,3}\left(q^{5 / 4},-q^{5 / 4},-q^{1 / 2},-1,-1\right)$. Using Corollary 2.17, we have

$$
\begin{aligned}
g_{3,5,3}\left(q^{5 / 4},-q^{5 / 4},-q^{1 / 2},-1,-1\right)=\left[j\left(q^{5 / 4} ;-q^{3 / 2}\right)+j\left(-q^{5 / 4} ;-q^{3 / 2}\right)\right] m\left(-q^{11}, q^{24},-1\right) \\
+q^{5 / 4}\left[j\left(-q^{15 / 4} ;-q^{3 / 2}\right)-j\left(q^{15 / 4} ;-q^{3 / 2}\right)\right] m\left(-q^{3}, q^{24},-1\right) \\
\quad-q^{4}\left[j\left(q^{25 / 4} ;-q^{3 / 2}\right)+j\left(q^{25 / 4} ;-q^{3 / 2}\right)\right] m\left(-q^{-5}, q^{24},-1\right) \\
=2 J_{2} m\left(-q^{11}, q^{24},-1\right)+2 q^{5} J_{12,6} m\left(-q^{3}, q^{24},-1\right) \\
\quad-2 q^{4} J_{14,6} m\left(-q^{-5}, q^{24},-1\right) \\
=2 J_{2} m\left(-q^{11}, q^{24},-1\right)-2 q^{-6} J_{2} m\left(-q^{-5}, q^{24},-1\right),
\end{aligned}
$$

where the second equality follows from applying $(2.1 \mathrm{f})$ to each bracketed term, and the last equality follows from (2.1a). Using (2.19d) and (2.19b)

$$
\begin{aligned}
g_{3,5,3} & \left(q^{5 / 4},-q^{5 / 4},-q^{1 / 2},-1,-1\right) \\
& =2 J_{2}\left(1+q^{-13} m\left(-q^{-13}, q^{24},-1\right)\right)+2 q^{-1} J_{2} m\left(-q^{5}, q^{24},-1\right) \\
& =2 J_{2}-2 J_{2} m\left(-q^{13}, q^{24},-1\right)+2 q^{-1} J_{2} m\left(-q^{5}, q^{24},-1\right) .
\end{aligned}
$$

Using Proposition 2.12 with $q \rightarrow q^{8}, x=-q, z=q^{-3}$,

$$
\begin{aligned}
-q g\left(-q, q^{8}\right) & =-m\left(-q^{13}, q^{24},-1\right)+q^{-1} m\left(-q^{5}, q^{24},-1\right)-\frac{q J_{8}^{2} j\left(-q^{-2} ; q^{8}\right) j\left(q^{-3} ; q^{24}\right)}{j\left(-q ; q^{8}\right) j\left(q^{-3} ; q^{8}\right) j\left(-1 ; q^{24}\right)} \\
& =-m\left(-q^{13}, q^{24},-1\right)+q^{-1} m\left(-q^{5}, q^{24},-1\right)-\frac{q^{-1} J_{8}^{2} \bar{J}_{2,8} J_{3,24}}{\bar{J}_{1,8} J_{3,8} \bar{J}_{0,24}} .
\end{aligned}
$$

So we have

$$
g_{3,5,3}\left(q^{5 / 4},-q^{5 / 4},-q^{1 / 2},-1,-1\right)=2 J_{2}-2 J_{2} q g\left(-q, q^{8}\right)+\frac{2 q^{-1} J_{8}^{2} J_{2} \bar{J}_{2,8} J_{3,24}}{\bar{J}_{1,8} J_{3,8} \bar{J}_{0,24}} .
$$


We compute $\theta_{3,2}\left(q^{5 / 4},-q^{5 / 4},-q^{1 / 2}\right)$. Using Corollary 2.17, we have

$$
\begin{aligned}
\theta_{3,2}\left(q^{5 / 4},-q^{5 / 4},-q^{1 / 2}\right)= & \frac{q^{-3 / 2}}{2 \bar{J}_{0,24}} \cdot \frac{j\left(q^{5} ; q^{4}\right) \bar{J}_{4,16}}{j\left(q^{5} ; q^{8}\right)^{2}} \\
& \cdot\left[j\left(q^{5 / 2} ; q^{4}\right)^{2} j\left(-q^{3 / 4} ;-q^{3 / 2}\right)-j\left(-q^{5 / 2} ; q^{4}\right)^{2} j\left(q^{3 / 4} ;-q^{3 / 2}\right)\right]
\end{aligned}
$$

Using (2.1b) and (2.1e $)$ yields

$$
j\left(-q^{3 / 4} ;-q^{3 / 2}\right)=j\left(q^{3 / 4} ;-q^{3 / 2}\right)=J_{3,6},
$$

so we can write

$$
\begin{aligned}
\theta_{3,2}\left(q^{5 / 4},-q^{5 / 4},-q^{1 / 2}\right)= & -\frac{q^{-5 / 2}}{2 \bar{J}_{0,24}} \cdot \frac{J_{1,4} \bar{J}_{4,16} J_{3,6}}{j\left(q^{5}, q^{8}\right)^{2}} \cdot\left[j\left(q^{5 / 2} ; q^{4}\right)^{2}-j\left(-q^{5 / 2} ; q^{4}\right)^{2}\right] \\
= & -\frac{q^{-5 / 2}}{2 \bar{J}_{0,24}} \cdot \frac{J_{1,4} \bar{J}_{4,16} J_{3,6}}{j\left(q^{5} ; q^{8}\right)^{2}} \\
& \cdot\left(j\left(q^{5 / 2} ; q^{4}\right)+j\left(-q^{5 / 2} ; q^{4}\right)\right) \cdot\left(j\left(q^{5 / 2} ; q^{4}\right)-j\left(-q^{5 / 2} ; q^{4}\right)\right) \\
= & \frac{q^{-5 / 2}}{2 \bar{J}_{0,24}} \cdot \frac{J_{1,4} \bar{J}_{4,16} J_{3,6}}{j\left(q^{5} ; q^{8}\right)^{2}} \cdot 2 j\left(-q^{9} ; q^{16}\right) \cdot 2 q^{5 / 2} j\left(-q^{17} ; q^{16}\right),
\end{aligned}
$$

where the last line follows from applying (2.1f) with $m=2$ to each expression in parentheses. Simplifying with elementary theta function properties gives

$$
\theta_{3,2}\left(q^{5 / 4},-q^{5 / 4},-q^{1 / 2}\right)=\frac{2 q^{-1} J_{3,6} J_{16} J_{8} J_{2,16}}{\bar{J}_{0,24} J_{5,8}} .
$$

So proving (1.11) is equivalent to showing

$$
\frac{2 q^{-1} J_{8}^{2} \bar{J}_{2,8} J_{3,24}}{\bar{J}_{1,8} J_{3,8} \bar{J}_{0,24}}-\frac{2 q^{-1} J_{3,6} J_{16} J_{8} J_{2,16}}{\bar{J}_{0,24} J_{5,8} J_{2}}=-\frac{J_{1,2} \bar{J}_{3,8}}{J_{2}} .
$$

But elementary theta function properties shows that (4.3) is equivalent to identity (2.10) of Proposition 2.6.

The proof of (1.12) is similar. Here we find that

$$
\begin{aligned}
\sum_{n=0}^{\infty} \frac{q^{2 n^{2}+2 n}}{(-q ; q)_{2 n+1}} & =\frac{1}{\left(q^{2} ; q^{2}\right)_{\infty}}\left(\sum_{\substack{n+j \geq 0 \\
n-j \geq 0}}-\sum_{\substack{n+j<0 \\
n-j<0}}\right)(-1)^{j} q^{4 n^{2}+3 n-j^{2}} \\
& =\frac{1}{J_{2}} \cdot f_{3,5,3}\left(q^{9 / 4},-q^{9 / 4},-q^{1 / 2}\right) .
\end{aligned}
$$

Using Corollary 2.17 and arguing as above reduces proving (1.12) to identity (2.11) of Proposition 2.6. 
ERIC MORTENSON

\section{Proof of Theorem 1.7}

Specializing Theorem 1.4, we have that

$$
\begin{aligned}
f_{4,4,1}(x, y, q)= & h_{4,4,1}(x, y, q,-1,-1) \\
& -\sum_{d=0}^{3} \frac{q^{3\left(\begin{array}{c}
d+1 \\
2
\end{array}\right)} j\left(q^{3+3 d} y ; q^{4}\right) j\left(-q^{9-3 d} x / y ; q^{12}\right) J_{12}^{3} j\left(-q^{9+3 d} / y^{3} ; q^{12}\right)}{\bar{J}_{0,3} \bar{J}_{0,12} j\left(-q^{6} x / y^{4} ; q^{12}\right) j\left(q^{3+3 d} y / x ; q^{12}\right)},
\end{aligned}
$$

where

$$
h_{4,4,1}(x, y, q,-1,-1)=j\left(x ; q^{4}\right) m\left(-q^{3} y / x, q^{3},-1\right)+j(y ; q) m\left(q^{6} x / y, q^{12},-1\right) .
$$

We prove identity (1.14). We first define

$$
\operatorname{sg}(r, s):=(\operatorname{sg}(r)+\operatorname{sg}(s)) / 2 .
$$

We start with the right-hand side of (1.14):

$$
\begin{aligned}
\sum_{n=0}^{\infty} & (-1)^{n} q^{2 n^{2}+n}\left(1+q^{2 n+1}\right) \sum_{j=-n}^{n}(-1)^{j} q^{-3 j^{2} / 2+j / 2} \\
& =\sum_{\substack{n \geq 0 \\
-n \leq j \leq n}}(-1)^{n+j} q^{2 n^{2}+n-3 j^{2} / 2+j / 2}+q \sum_{\substack{n \geq 0 \\
-n \leq j \leq n}}(-1)^{n+j} q^{2 n^{2}+3 n-3 j^{2} / 2+j / 2} \\
& =\sum_{n, j} \operatorname{sg}(j, n-j)(-1)^{n+j} q^{2 n^{2}+n-3 j^{2} / 2+j / 2}+q \sum_{n, j} \operatorname{sg}(j, n-j)(-1)^{n+j} q^{2 n^{2}+3 n-3 j^{2} / 2+j / 2} \\
& =f_{4,4,1}\left(q^{3},-q^{2}, q\right)+q f_{4,4,1}\left(q^{5},-q^{4}, q\right),
\end{aligned}
$$

where the last line follows from the substitutions $u=j, v=n-j$.

We first consider the Appell-Lerch sum expresssion. Using (5.2), we have

$$
\begin{aligned}
h_{4,4,1}\left(q^{3},-q^{2}, q\right) & +q h_{4,4,1}\left(q^{5},-q^{4}, q\right)=j\left(q^{3} ; q^{4}\right) m\left(q^{2}, q^{3},-1\right)+j\left(-q^{2} ; q\right) m\left(q, q^{12},-1\right) \\
& +q j\left(q^{5} ; q^{4}\right) m\left(q^{2}, q^{3},-1\right)+q j\left(-q^{4} ; q\right) m\left(q^{-5}, q^{12},-1\right) \\
= & 2 \bar{J}_{1,4} m\left(q^{5}, q^{12},-1\right)+2 q^{-1} \bar{J}_{1,4} m\left(q, q^{12},-1\right)
\end{aligned}
$$

where the last line follows from (2.1a) and (2.19b). Thus

$$
\begin{aligned}
f_{4,4,1}\left(q^{3},-q^{2}, q\right)+q f_{4,4,1} & \left(q^{5},-q^{4}, q\right)=2 \bar{J}_{1,4} m\left(q^{5}, q^{12},-1\right)+2 q^{-1} \bar{J}_{1,4} m\left(q, q^{12},-1\right) \\
& -\sum_{d=0}^{3} \frac{q^{3\left(\begin{array}{c}
d+1 \\
2
\end{array}\right)} j\left(-q^{5+3 d} ; q^{4}\right) j\left(q^{10-3 d} ; q^{12}\right) J_{12}^{3} j\left(q^{3+3 d} ; q^{12}\right)}{\bar{J}_{0,3} \bar{J}_{0,12} j\left(-q ; q^{12}\right) j\left(-q^{2+3 d} ; q^{12}\right)} \\
& -q \sum_{d=0}^{3} \frac{q^{3\left(\begin{array}{c}
d+1 \\
2
\end{array}\right)} j\left(-q^{7+3 d} ; q^{4}\right) j\left(q^{10-3 d} ; q^{12}\right) J_{12}^{3} j\left(q^{-3+3 d} ; q^{12}\right)}{\bar{J}_{0,3} \bar{J}_{0,12} j\left(-q^{-5} ; q^{12}\right) j\left(-q^{2+3 d} ; q^{12}\right)}
\end{aligned}
$$


Using (2.19f) and grouping terms, we can rewrite (5.5) as

$$
\begin{gathered}
f_{4,4,1}\left(q^{3},-q^{2}, q\right)+q f_{4,4,1}\left(q^{5},-q^{4}, q\right) \\
=\bar{J}_{1,4} m\left(q^{5}, q^{12}, q^{4}\right)+\bar{J}_{1,4} m\left(q^{5}, q^{12}, q^{8}\right)+q^{-1} \bar{J}_{1,4} m\left(q, q^{12}, q^{4}\right)+q^{-1} \bar{J}_{1,4} m\left(q, q^{12}, q^{8}\right) \\
+\frac{\bar{J}_{1,4} J_{12}^{3} \bar{J}_{4,12}}{\bar{J}_{0,12} \bar{J}_{5,12} J_{4,12}}\left[\frac{\bar{J}_{9,12}}{J_{9,12}}-\frac{\bar{J}_{1,12}}{J_{1,12}}\right]+q^{-1} \frac{\bar{J}_{1,4} J_{12}^{3} \bar{J}_{4,12}}{\bar{J}_{0,12} \bar{J}_{1,12} J_{4,12}}\left[\frac{\bar{J}_{5,12}}{J_{5,12}}+\frac{\bar{J}_{9,12}}{J_{9,12}}\right] \\
-\frac{1}{\bar{J}_{0,3} \bar{J}_{0,12}}\left(q^{-1} \frac{\bar{J}_{1,4} J_{12}^{3} J_{3,12}}{\bar{J}_{1,12}}\left[\frac{J_{2,12}}{\bar{J}_{2,12}}+\frac{J_{4,12}}{\bar{J}_{4,12}}\right]+\frac{\bar{J}_{1,4} J_{12}^{3} J_{3,12}}{\bar{J}_{5,12}}\left[\frac{J_{4,12}}{\bar{J}_{4,12}}-\frac{J_{2,12}}{\bar{J}_{2,12}}\right]\right. \\
\left.\quad+\frac{\bar{J}_{0,4} J_{12}^{3} J_{6,12}}{\bar{J}_{1,12} \bar{J}_{5,12}}\left[J_{1,12}+q^{-1} J_{7,12}\right]\right) .
\end{gathered}
$$

With (2.44) in mind, to prove (1.14) it remains to show that the sum of quotients of theta functions in (5.6) is zero. Using identities (2.3c), (2.3d), and (2.1f), the bracketed expressions can be evaluated and the terms can then be rearranged to show that the sum of quotients of theta functions is

$$
\begin{aligned}
& 2 q^{-1} \frac{\bar{J}_{1,4} J_{12}^{3} \bar{J}_{4,12} J_{8,24}}{\bar{J}_{0,12} J_{3,12} J_{4,12}} \cdot\left[\frac{J_{14,24}}{\bar{J}_{1,12} J_{5,12}}-q^{2} \frac{J_{2,24}}{\bar{J}_{5,12} J_{1,12}}\right] \\
& -2 q^{-1} \frac{\bar{J}_{1,4} J_{12}^{3} J_{3,12} J_{6,24}}{\bar{J}_{0,3} \bar{J}_{0,12} \bar{J}_{2,12} \bar{J}_{4,12}} \cdot\left[\frac{J_{14,24}}{\bar{J}_{1,12}}+q^{3} \frac{J_{2,24}}{\bar{J}_{5,12}}\right]-\frac{\bar{J}_{0,4} J_{12}^{3} J_{6,12}}{\bar{J}_{0,3} \bar{J}_{0,12} \bar{J}_{1,12} \bar{J}_{5,12}} \cdot j\left(-q ;-q^{3}\right) \\
& =2 q^{-1} \frac{\bar{J}_{1,4} J_{12}^{3} \bar{J}_{4,12} J_{8,24}}{\bar{J}_{0,12} J_{3,12} J_{4,12}} \cdot \frac{J_{24}}{J_{12}^{2}} \cdot \frac{J_{1,3} \bar{J}_{1,3}}{\bar{J}_{1,12} \bar{J}_{5,12}} \\
& -2 q^{-1} \frac{\bar{J}_{1,4} J_{12}^{3} J_{3,12} J_{6,24}}{\bar{J}_{0,3} \bar{J}_{0,12} \bar{J}_{2,12} \bar{J}_{4,12}} \cdot \frac{1}{\bar{J}_{1,12} \bar{J}_{5,12}} \cdot \frac{\bar{J}_{3,12} J_{8} \bar{J}_{4,12}}{\bar{J}_{6,24}} \\
& -\frac{\bar{J}_{0,4} J_{12}^{3} J_{6,12}}{\bar{J}_{0,3} \bar{J}_{0,12} \bar{J}_{1,12} \bar{J}_{5,12}} \cdot j\left(-q ;-q^{3}\right) \text {, }
\end{aligned}
$$

where the first bracketed expression was evaluated with (2.1g) and (2.1f), and the second bracketed expression was evaluated with Proposition 2.4 with $q \rightarrow-q^{3}$ and $x \rightarrow-q^{-1}$. Using a straightforward but lengthly argument with (2.1g) and (2.1e), showing that the right-hand side of (5.7) is zero is equivalent to showing

$$
2 J_{12}^{2} J_{24}^{2}-J_{6,24} \bar{J}_{6,24} J_{12} J_{24}-J_{6,24}^{2} \bar{J}_{6,24}^{2}=0,
$$

which is straightforward.

We prove identity (1.15). We start with the right-hand side of (1.15):

$$
\sum_{n=0}^{\infty}(-1)^{n} q^{2 n^{2}+2 n} \sum_{j=-n}^{n}(-1)^{j} q^{-3 j^{2} / 2+j / 2}=\sum_{\substack{n \geq 0 \\-n \leq j \leq n}}(-1)^{n+j} q^{2 n^{2}+2 n-3 j^{2} / 2+j / 2}
$$




$$
\begin{aligned}
& =\sum_{n, j} \operatorname{sg}(j, n-j)(-1)^{n+j} q^{2 n^{2}+2 n-3 j^{2} / 2+j / 2} \\
& =f_{4,4,1}\left(q^{4},-q^{3}, q\right)
\end{aligned}
$$

where the last line follows from the substitutions $u=j, v=n-j$. We first consider the Appell-Lerch sum expression. Using (5.2) we have

$h_{4,4,1}(x, y, q)=j\left(q^{4} ; q^{4}\right) m\left(q^{2}, q^{3},-1\right)+j\left(-q^{3} ; q\right) m\left(q^{-2}, q^{12},-1\right)=2 q^{-1} \bar{J}_{1,4} m\left(q^{2}, q^{12}-1\right)$.

Thus

$$
\begin{aligned}
f_{4,4,1}\left(q^{4},-q^{3}, q\right)= & 2 q^{-1} m\left(q^{2}, q^{12}-1\right) \\
& -\sum_{d=0}^{3} \frac{q^{3\left(\begin{array}{c}
d+1 \\
2
\end{array}\right)} j\left(-q^{6+3 d} ; q^{4}\right) j\left(q^{10-3 d} ; q^{12}\right) J_{12}^{3} j\left(q^{3 d} ; q^{12}\right)}{\bar{J}_{0,3} \bar{J}_{0,12} j\left(-q^{-2} ; q^{12}\right) j\left(-q^{2+3 d} ; q^{12}\right)}
\end{aligned}
$$

Using (2.19f) and grouping terms, we can rewrite (5.10) as

$$
\begin{aligned}
f_{4,4,1}\left(q^{4},\right. & \left.-q^{3}, q\right)=q^{-1} m\left(q^{2}, q^{12},-q^{3}\right)+q^{-1} m\left(q^{2}, q^{12},-q^{9}\right) \\
& +q^{-1} \frac{\bar{J}_{1,4} J_{12}^{3} J_{3,12}}{\bar{J}_{0,12} \bar{J}_{2,12} \bar{J}_{3,12}} \cdot\left[\frac{J_{5,12}}{\bar{J}_{5,12}}+\frac{J_{11,12}}{\bar{J}_{11,12}}\right] \\
& -\frac{1}{\bar{J}_{0,3} \bar{J}_{0,12}} \cdot\left(q^{-1} \frac{\bar{J}_{1,4} J_{12}^{3} J_{3,12}}{\bar{J}_{2,12}} \cdot\left[\frac{J_{5,12}}{\bar{J}_{5,12}}+\frac{J_{11,12}}{\bar{J}_{11,12}}\right]+q^{-1} \frac{\bar{J}_{0,4} J_{4,12} J_{12}^{3} J_{6,12}}{\bar{J}_{2,12} \bar{J}_{8,12}}\right)
\end{aligned}
$$

With (2.43) in mind, to prove (1.15) it remains to show that the sum of quotients of theta functions in (5.11) is zero. Using identity (2.3d), the bracketed expression can be evaluated and the terms can then be rearranged to show that the sum of quotients of theta functions in (5.11) is

$$
\begin{aligned}
& q^{-1} \frac{\bar{J}_{1,4} J_{12}^{3} J_{3,12}}{\bar{J}_{0,12} \bar{J}_{2,12} \bar{J}_{3,12}} \cdot \frac{2 J_{16,24} J_{18,24}}{\bar{J}_{5,12} \bar{J}_{11,12}} \\
& \quad-\frac{1}{\bar{J}_{0,3} \bar{J}_{0,12}} \cdot\left(q^{-1} \frac{\bar{J}_{1,4} J_{12}^{3} J_{3,12}}{\bar{J}_{2,12}} \cdot \frac{2 J_{16,24} J_{18,24}}{\bar{J}_{5,12} \bar{J}_{1,12}}+q^{-1} \frac{\bar{J}_{0,4} J_{4,12} J_{12}^{3} J_{6,12}}{\bar{J}_{2,12} \bar{J}_{8,12}}\right) .
\end{aligned}
$$

Using the identity $\bar{J}_{3,12}=2 \bar{J}_{0,3}$, and elementary theta function properties, it is straightforward to verify that the right-hand side of (5.12) is equal to zero.

We prove identity (1.16). Here we use the Bailey pair

$$
B_{n}^{\prime}(0, q):=1, \quad A_{n}^{\prime}\left(q^{2}, 0, q\right):=\frac{q^{2 n^{2}+3 n}\left(1-q^{2 n+2}\right)}{(1-q)\left(1-q^{2}\right)} \sum_{j=-n-1}^{n}(-1)^{j} q^{-j(3 j+5) / 2}
$$


from Theorem 4 and Lemma 6 of [3]. Bailey's lemma [3, (2.4)] with $q \rightarrow q^{2}, \rho_{1}=-q^{3}$, $\rho_{2}=-q^{2}$, and $a=q^{4}$, then gives

$$
\begin{aligned}
& J_{1,2} \overline{\phi_{0}}(q)=\sum_{n=0}^{\infty} q^{4 n^{2}+7 n}\left(1-q^{2 n+2}\right) \sum_{j=-n-1}^{n}(-1)^{j} q^{-3 j^{2}-5 j} \\
&=\left(\sum_{\substack{r, s \geq 0 \\
r \neq s}}-\sum_{\substack{r, s<0 \\
(\bmod 2)}}\right)(-1)^{(r-s-1) / 2} q^{(r+s+1)^{2} / 4+(3 r+2) s-1}, \\
& \substack{\bmod 2)}
\end{aligned}
$$

which is what one finds in [7, (2.7)]. Replacing $(r, s)$ with $(2 R+1,2 S)$ and $(2 R, 2 S+1)$, we obtain

$$
\begin{aligned}
J_{1,2} \overline{\phi_{0}}(q) & =f_{1,7,1}\left(q^{3}, q^{13}, q^{2}\right)-q^{2} f_{1,7,1}\left(q^{9}, q^{7}, q^{2}\right) \\
& =-q^{2} f_{1,7,1}\left(q^{15}, q^{5}, q^{2}\right)+q^{4} f_{1,7,1}\left(q^{9}, q^{11}, q^{2}\right) \\
& =q^{-1} f_{1,7,1}\left(q, q^{3}, q^{2}\right)-q^{-1} J_{1,2}+q^{4} f_{1,7,1}\left(q^{9}, q^{11}, q^{2}\right) \\
& =q^{-1} \sum_{n=0}^{\infty} q^{4 n^{2}+n}\left(1-q^{6 n+3}\right) \sum_{j=-n}^{n}(-1)^{j} q^{-3 j^{2}-j}-q^{-1} J_{1,2} .
\end{aligned}
$$

We prove identity (1.17). Here we use the Bailey pair

$$
B_{n}^{\prime}(0, q):=1, \quad A_{n}^{\prime}(q, 0, q):=\frac{q^{2 n^{2}+n}\left(1-q^{2 n+1}\right)}{1-q} \sum_{j=-n}^{n}(-1)^{j} q^{-j(3 j+1) / 2}
$$

from Theorem 4 and Lemma 7 of [3]. Bailey's lemma [3, (2.4)] with $q \rightarrow q^{2}, \rho_{1}=-q$, $\rho_{2}=-q^{2}$, and $a=q^{2}$, then gives the result.

\section{Proof of Theorem 1.8}

We prove (1.20); the proof of (1.21) is similar. We have

$$
\begin{aligned}
2 q^{2} \bar{\phi}_{0}\left(q^{2}\right) & =\psi(q)+\psi(-q) & & (\text { by }(\underline{1.18})) \\
& =q g\left(q, q^{4}\right)-q g\left(-q, q^{4}\right) & & (\text { by }(\underline{2.42})) \\
& =-2+2 q^{2} g\left(-q^{2}, q^{16}\right)+2 \cdot \frac{J_{8} \bar{J}_{4,16}^{2}}{J_{2,8} \bar{J}_{14,16}} . & & \text { (by (2.26) })
\end{aligned}
$$

Elementary theta function properties gives the result.

\section{ACKNOWLEDGEMENTS}

We would like to thank Dean Hickerson and the referee for helpful comments and suggestions. 


\section{REFERENCES}

[1] M. P. Appell, Sur les fonctions doublement périodiques de troisième espèce, Annales scientifiques de l'ENS, 3e série, t. I, p. 135, t. II, p. 9, t. III, p. 9, 1884-1886.

[2] G. E. Andrews, Hecke modular forms and the Kac-Peterson identities, Trans. Amer. Math. Soc., 283, (1984), pp. 451-458.

[3] G. E. Andrews, The fifth and seventh order mock theta functions, Trans. Amer. Math. Soc., 293, (1986), pp. 113-134.

[4] G. E. Andrews, q-orthogonal polynomials, Rogers-Ramanujan identities, and mock theta functions, Proceedings of the Steklov Institute of Mathematics, to appear.

[5] G. E. Andrews, B. C. Berndt, Ramanujan's Lost Notebook Part I, Springer, New York, 2005.

[6] A. O. L. Atkin, P. Swinnerton-Dyer, Some properties of partitions, Proc. London Math. Soc. (3), 4 (1954), pp. 84-106.

[7] K. Bringmann, K. Hikami, J. Lovejoy, On the modularity of the unified WRT invariants of certain Seifert manifolds, Adv. Appl. Math., 46 (2011), pp. 86-93.

[8] D. Hickerson, A proof of the mock theta conjectures, Invent. Math., 94 (1988), no. 3, pp. 639-660.

[9] D. Hickerson, On the seventh order mock theta functions, Invent. Math., 94 (1988), no. 3, pp. 661-677.

[10] D. Hickerson, E. Mortenson Hecke-type double sums, Appell-Lerch sums, and mock theta functions (I), submitted.

[11] V. Kac, D. Peterson, Infinite-dimensional Lie algebras, theta functions and modular forms, Adv. in Math., 53 (1984), no. 2, pp. 125-264.

[12] M. Lerch, Poznámky $k$ theorii funkci elliptických, Rozpravy České Akademie Císaře Františka Josefa pro vědy, slovesnost a umění v praze, 24, (1892), pp. 465-480.

[13] M. Lerch, Bemerkungen zur Theorie der elliptischen Funktionen, Jahrbuch über die Fortschritte der Mathematik, 24, (1892), pp. 442-445.

[14] S. Ramanujan, The Lost Notebook and Other Unpublished Papers, Narosa Publishing House, New Delhi, 1988.

[15] G. N. Watson, The final problem: an account of the mock theta functions, J. London Math. Soc., 11 (1936), pp. 55-80.

[16] S. P. Zwegers, Mock theta functions, Ph.D. Thesis, Universiteit Utrecht, 2002.

Department of Mathematics, The University of Queensland, Brisbane, Australia 4072

E-mail address: mort@maths.uq.edu.au 